\title{
Extragalactic Transients in the Era of Wide-Field Radio Surveys. I. Detection Rates and Light Curve Characteristics
}

\section{Citation}

Metzger, Brian D., P. K. G. Williams, and Edo Berger. 2015. Extragalactic Transients in the Era of Wide-Field Radio Surveys. I. Detection Rates and Light Curve Characteristics. The Astrophysical Journal 806, no. 2: 224. doi:10.1088/0004-637x/806/2/224.

\section{Published Version}

doi:10.1088/0004-637X/806/2/224

\section{Permanent link}

http://nrs.harvard.edu/urn-3:HUL.InstRepos:30498355

\section{Terms of Use}

This article was downloaded from Harvard University's DASH repository, and is made available under the terms and conditions applicable to Open Access Policy Articles, as set forth at http:// nrs.harvard.edu/urn-3:HUL.InstRepos:dash.current.terms-of-use\#OAP

\section{Share Your Story}

The Harvard community has made this article openly available.

Please share how this access benefits you. Submit a story.

\section{Accessibility}


Draft VERsion FEBRUARY 6, 2015

Preprint typeset using LATEX style emulateapj v. 08/22/09

\title{
EXTRAGALACTIC TRANSIENTS IN THE ERA OF WIDE-FIELD RADIO SURVEYS. I. DETECTION RATES AND LIGHT CURVE CHARACTERISTICS
}

\author{
Brian D. Metzger ${ }^{1}$, P. K. G. Williams ${ }^{2}$, \& Edo Berger ${ }^{2}$ \\ Draft version February 6, 2015
}

\begin{abstract}
The impending era of wide-field radio surveys has the potential to revolutionize our understanding of astrophysical transients. Here we evaluate the prospects of a wide range of planned and hypothetical radio surveys using the properties and volumetric rates of known and hypothetical classes of extragalactic synchrotron radio transients (e.g., on- and off-axis gamma-ray bursts, supernovae, tidal disruption events, compact object mergers). Utilizing these sources and physically motivated considerations we assess the allowed phase-space of radio luminosity and peak timescale for extragalactic transients. We also include for the first time effects such as redshift evolution of the rates, K-corrections, and non-Euclidean luminosity distance, which affect the detection rates of the most sensitive surveys. The number of detected events is calculated by means of a Monte Carlo method, using the various survey properties (depth, cadence, area) and realistic detection criteria that include a cut on the minimum variability of the transients during the survey and an assessment of host galaxy contamination. For the detected events we also quantify how well each light curve is characterized (e.g., the fraction of sources with a measured rise time, decline time, and peak brightness). We find that near-term GHz frequency surveys (ASKAP/VAST, Very Large Array Sky Survey) will detect few events: $\lesssim 30-50$ on- and off-axis long GRBs and off-axis tidal disruption events, and $\sim 10-20$ neutron star binary mergers if $\sim 1 \%$ of the mergers result in a stable millisecond magnetar. Lowfrequency surveys (e.g., LOFAR) are unlikely to detect any transients, while a hypothetical large-scale mm survey may detect $\sim 40$ on-axis long GRBs. On the other hand, we find that SKA surveys at $\sim 0.1-1 \mathrm{GHz}$ have the potential to uncover thousands of transients, mainly on- and off-axis long GRBs, on-axis short GRBs, off-axis TDEs, and neutron star binary mergers with magnetar remnants. Subject headings: gamma-ray bursts: general — radio continuum: general — supernovae: general — surveys
\end{abstract}

\section{INTRODUCTION}

Radio astronomy appears poised for a revolution in the study of transient phenomena thanks to the advent of wide-field interferometers. At meter wavelengths (tens to hundreds of $\mathrm{MHz}$ ) it is now feasible to image the full primary beams of dipole antennae, resulting in new advanced arrays such as the LOw Frequency ARray (LOFAR; van Haarlem et al. 2013), the Murchison Widefield Array (MWA; Lonsdale et al. 2009) and the Long Wavelength Array (LWA; Ellingson et al. 2009). At centimeter wavelengths ( $\mathrm{GHz}$ frequencies) a new generation of widefield facilities is also being developed, such as MeerKAT (Booth et al. 2009) and the Australian Square Kilometer Array Pathfinder (ASKAP; Johnston et al. 2008a), and existing facilities are undergoing upgrades, including the Apertif project at the Westerbork Synthesis Radio Telescope (WSRT; Oosterloo et al. 2009) and the expansion of the Karl G. Jansky Very Large Array (VLA; Perley et al. 2011). The VLA upgrade makes it possible to reach greater image depth in a shorter time, raising the possibility of conducting a new survey combining the sensitivity of the FIRST survey (Becker et al. 1995) with the large sky footprint of the NVSS survey (Condon et al. 1998). These telescopes and new technologies are ulti-

\footnotetext{
${ }^{1}$ Columbia Astrophysics Laboratory, Pupin Hall, New York, NY, 10027, USA; bmetzger@phys.columbia.edu

${ }^{2}$ Harvard-Smithsonian Center for Astrophysics, 60 Garden Street, Cambridge, MA 02138; pwilliams@cfa.harvard.edu, eberger@cfa.harvard.edu
}

mately paving the way for the Square Kilometer Array (SKA; Carilli \& Rawlings 2004), which will further transform radio astronomy with its unprecedented scale and sensitivity.

Despite the overall maturity of radio astronomy, surprisingly little is known about what astrophysical sources might dominate the transient radio sky. At long wavelengths, the brightest sources are expected to result from coherent emission processes. Examples include 'giant pulses' from Galactic pulsars (e.g. Jessner et al. 2005) and cyclotron maser emission from brown dwarfs and possibly exoplanets (Berger 2002; Hallinan et al. 2008); brighter extra-galactic analogs of such events are speculated to accompany rare violent events, such as giant magnetar flares (Lyubarsky 2014) and mergers of binary neutron stars (Hansen \& Lyutikov 2001), and may be connected to the fast radio burst (FRB) phenomenon (e.g., Thornton et al. 2013; Spitler et al. 2014). At centimeter wavelengths, and on longer timescales, synchrotron sources are expected to dominate the transient sky. These are generally powered by the shock interaction between fast ejecta from an energetic explosion and dense ambient gas, as in the case of supernovae ( $\mathrm{SNe}$ ) and gamma-ray bursts (GRBs). Slow-timescale Galactic radio transients are expected to be dominated by a different population, such as flares from $\mathrm{M}$ dwarfs and X-ray binaries, with rates that are believed to be comparable to those of extragalactic events (Williams et al. 2013).

In this work, we consider and study extragalactic syn- 
chrotron transients. These are the best understood events based on radio follow-up of discoveries at other wavelengths, they are of proven astrophysical interest, and they are amenable to detection by imaging surveys, especially those operating at centimeter wavelengths. Known examples include core-collapse SNe (Kulkarni et al. 1998; Weiler et al. 2002; Berger et al. 2003; Soderberg et al. 2010), GRB afterglows (Frail et al. 1997; Chandra \& Frail 2012), and relativistic jets from tidal disruption events (Giannios \& Metzger 2011; Zauderer et al. 2011; Berger et al. 2012; Zauderer et al. 2013).

Searches for these and related types of radio transients help to motivate the science cases for all of the telescopes and surveys mentioned above. With these next generation projects coming online in the near future, it is essential to consider how many and which kinds of transient events will be detected, and how survey strategies may be optimized to answer particular science questions. Most untargeted surveys to date have detected few if any events (e.g., Carilli et al. 2003; Croft et al. 2010a; Bell et al. 2011), and despite careful vetting, a substantial number of detected candidates have turned out to be instrumental artifacts (Gal-Yam et al. 2006; Ofek et al. 2010; Croft et al. 2011; Frail et al. 2012). Thus, most of what is known empirically about the transient radio sky consists of upper limits. Complicating matters is the fact that these upper limits are often difficult to characterize and interpret, because the expected yield of a given survey results from a complex interplay between the properties of the transient events in question and the survey strategy. While this interplay is often considered in the derivation of upper limits and event rates (Bower et al. 2007; Croft et al. 2011; Mooley et al. 2013; Williams et al. 2013), it is challenging to harmonize the results from different studies for inter-comparison, or to extrapolate them to new surveys.

While the current results from untargeted surveys are of limited use for predicting the yields of the nextgeneration projects, all is not lost since some information is available from radio follow-up observations and from generic theoretical considerations. The volumetric rates of some of the expected events have been explored in detail (e.g., Wang \& Merritt 2004; Guetta \& Della Valle 2007; Wanderman \& Piran 2010; Kim et al. 2013), and targeted radio follow-up of transients triggered from other wavelengths has yielded significant insight into the radio properties of some of the event classes mentioned above. In other cases, detailed numerical models allow predictions of radio emission that are well-motivated by both observations and theory (e.g., Nakar \& Piran 2011; van Eerten \& MacFadyen 2011).

Despite this available knowledge, the predicted rates of radio transient occurrence and detection - taking into account the expected luminosities and durations - have generally not received detailed theoretical attention. Frail et al. (2012) estimated event rates ignoring cosmological effects such as non-Euclidean luminosity distances, K-corrections, and time dilation, instead assuming the standard isotropic Euclidean scaling $N_{\text {all-sky }}\left(>F_{\nu}\right) \propto F_{\nu}^{-3 / 2}$, where $N_{\text {all-sky }}$ is the instantaneous number of events on the sky brighter than a given flux density, $F_{\nu}$. Other works considered specific event classes with more detailed analyses (Ghirlanda et al. 2014; Feng et al. 2014) but do not treat the surveys themselves in detail. As we show in this work, the interplay between the transient luminosities and timescales, the survey cadence, and the total survey duration is critical to determining the yield of a given survey, and the ability to separate transient from steady sources.

Here we offer the first detailed and comprehensive assessment of the discovery prospects for extragalactic synchrotron radio transients in the upcoming wide-field survey era. We do so by considering multiple effects: the specific observing strategies of several proposed and hypothetical surveys, the light curves for a wide range of known and theoretical transients spanning a broad range of energies, collimation, and ambient densities (and hence timescales and luminosities), a judicious choice of detectability metrics, and realistic volumetric rates and their cosmological evolution. Our goal is to provide well-motivated estimates for what various surveys will actually discover using the best information currently available. Future radio surveys also have the potential to discover new classes of transients beyond the ones we consider here. However, any transient source powered by synchrotron radiation from a rapidly expanding blastwave obeys fundamental physical constraints, such as the relation between peak luminosity and timescale, and a characteristic evolution from high to low frequencies. Thus, our investigation of a broad range of the synchrotron transient parameter space sets meaningful bounds on the possible detection rates even of event classes that we do not explicitly consider.

Our primary outputs in this paper are the numbers and basic light curve characteristics of detected transients as a function of event class and survey. In addition to this information it is also critical to consider what can (and cannot) be learned and extracted from each event after detection, such as the energy scale, collimation, ambient density, and nature of the transient, These will depend in part on the ability to robustly measure the peak luminosity, characteristic timescale, and redshift from a host galaxy. In a follow-up paper ("Paper II") we will investigate this topic, combining the radio light curves produced by our simulations from this paper with synchrotron models and realistic follow-up strategies. Our analysis will be conducted with an eye towards optimizing future transient surveys to yield not just detections but also astrophysical insight.

The paper is organized as follows. We begin by considering the general characteristics of radio emission from extragalactic synchrotron transients ( $\$ 2.1)$, and establishing the particular properties of the events that we consider in this paper, including luminosities, timescales, and volumetric rates $(\S 2.2)$. In $\S 3$ we describe the characteristics of the radio surveys that we simulate. Our Monte Carlo method and detection criteria for the simulations are described in $\S 4$, and their results are summarized in $\S 5$. We discuss the implications of our results and present our conclusions in $\S 6$.

\section{EXTRAGALACTIC TRANSIENTS}

In this work we focus on extragalactic synchrotronemitting transients, powered by shock interaction between fast ejecta and the ambient medium. The production of synchrotron emission in such transients is generic, and has been observed from various sources (e.g., GRBs, 
TABLE 1

Extragalactic Transient Classes

\begin{tabular}{|c|c|c|c|c|c|c|c|c|c|}
\hline Transient & $\begin{array}{c}\mathcal{R}(z=0) \\
\left(\mathrm{Gpc}^{-3} \mathrm{yr}^{-1}\right)\end{array}$ & $\begin{array}{c}E_{K} \\
(\mathrm{erg})\end{array}$ & $\begin{array}{c}n \\
\left(\mathrm{~cm}^{-3}\right)\end{array}$ & $\beta_{i}$ & $\begin{array}{c}\tau_{0.15}{ }^{(a)} \\
(\mathrm{d})\end{array}$ & $\begin{array}{c}\tau_{1.3}{ }^{(b)} \\
(\mathrm{d})\end{array}$ & $\begin{array}{c}\tau_{3}(c) \\
(\mathrm{d})\end{array}$ & $\begin{array}{c}\tau_{150}{ }^{(d)} \\
(\mathrm{d})\end{array}$ & Ref \\
\hline LGRB, $\theta_{\text {obs }}=0.2$ & $0.3^{\dagger}$ & $10^{51}$ & 1 & 1 & 320 & 62 & 31 & 4 & 1 \\
\hline $\mathrm{LGRB}, \theta_{\mathrm{obs}}=0.4$ & $1^{\dagger}$ & $10^{51}$ & 1 & 1 & 450 & 90 & 55 & 12 & 1 \\
\hline LGRB, $\theta_{\text {obs }}=0.8$ & $4^{\dagger}$ & $10^{51}$ & 1 & 1 & 1900 & 230 & 150 & 150 & 1 \\
\hline $\mathrm{LGRB}, \theta_{\mathrm{obs}}=1.57$ & $12^{\dagger}$ & $10^{51}$ & 1 & 1 & 1300 & 620 & 550 & 590 & 1 \\
\hline Low Luminosity LGRB ("LLGRB") & $500^{\dagger}$ & $10^{49}$ & 1 & 0.8 & 200 & 43 & 90 & 110 & 9 \\
\hline $\mathrm{SGRB}, \theta_{\mathrm{obs}}=0.2$ & $5^{\dagger}$ & $10^{50}$ & $10^{-3}$ & 1 & 220 & 110 & 90 & 110 & 6 \\
\hline $\mathrm{SGRB}, \theta_{\mathrm{obs}}=0.4$ & $15^{\dagger}$ & $10^{50}$ & $10^{-3}$ & 1 & 360 & 180 & 160 & 180 & 6 \\
\hline $\mathrm{SGRB}, \theta_{\mathrm{obs}}=0.8$ & $60^{\dagger}$ & $10^{50}$ & $10^{-3}$ & 1 & 730 & 480 & 410 & 480 & 6 \\
\hline $\mathrm{SGRB}, \theta_{\mathrm{obs}}=1.57$ & $185^{\dagger}$ & $10^{50}$ & $10^{-3}$ & 1 & 1900 & 2200 & 2000 & 650 & 6 \\
\hline On-Axis TDE ("Sw J1644+57") & $0.01^{\ddagger}$ & $10^{52}$ & 0.1 & 1 & 3700 & 920 & 1040 & 180 & $2-4$ \\
\hline Off-Axis TDE, spherical & $1^{\ddagger}$ & $10^{52}$ & 0.1 & 0.8 & 3700 & 900 & 900 & 900 & 5 \\
\hline NSM: prompt BH & $500^{\dagger}$ & $3 \times 10^{50}$ & 0.1 & 0.2 & 4000 & 4000 & 4000 & 4000 & 7 \\
\hline NSM: stable remnant ("NSM-magnetar") & $5^{\dagger}$ & $3 \times 10^{52}$ & 0.1 & 1 & 2800 & 1300 & 1300 & 1300 & 8 \\
\hline Type Ib/c SNe ("RSN") & $5000^{\dagger}$ & $10^{47}$ & 1 & 0.2 & 870 & 120 & 55 & 1.1 & 10 \\
\hline
\end{tabular}

Noте. _ ${ }^{\dagger}$ Scaled with redshift according to star formation rate (Cucciati et al. 2012). $\ddagger$ Scaled with redshift according to volumetric density of supermassive black holes (Sijacki et al. 2014). (a) Light curve duration at observer frequency $\nu=0.15$ GHz. (b) Light curve duration at observer frequency $\nu=1.3 \mathrm{GHz} .{ }^{(c)}$ Light curve duration at observer frequency $\nu=3 \mathrm{GHz}$. $(d)$ Light curve duration at observer frequency $\nu=150 \mathrm{GHz}$. References: (1) van Eerten et al. 2010; (2) Zauderer et al. 2011; (3) Berger et al. 2012; (4) Zauderer et al. 2013; (5) Giannios \& Metzger 2011; (6) van Eerten \& MacFadyen 2011; (7) Nakar \& Piran 2011; (8) Metzger \& Bower 2014; (9) Barniol Duran et al. 2014; (10) Soderberg et al. 2008

SNe, TDEs). Here we consider a wide range of transients in terms of their energy scale and ambient density that include both relativistic and non-relativistic explosions, collimated and spherical outflows, with on-axis and offaxis orientations. As a result, these transients span a broad range of the luminosity-timescale phase-space that is commonly used to map radio transients; see Figure 1. We note that some of these transients have been extensively observed in the past using radio follow-up of discoveries at other wavelengths (e.g., on-axis GRBs, SNe), some include only a few known examples (e.g., TDEs), some are robustly predicted but have not been directly observed to date (e.g., binary neutron star mergers, offaxis GRBs), and some are purely hypothetical (e.g., neutron star mergers giving rise to a stable millisecond magnetar). While other types of extragalactic synchrotron radio transients could be hypothesized, the sources considered here span a sufficiently broad range of properties and volumetric rates to encompass future predictions.

\subsection{General Considerations of Timescale and Luminosity for Extragalactic Radio Transients}

For the purpose of placing general constraints on the light curves of extragalactic synchrotron radio transients we begin by considering the ejection of material with a kinetic energy of $E_{K}=10^{51} E_{K, 51}$ erg and an initial velocity of $v_{i}=\beta_{i} c$ (corresponding to an initial Lorentz factor of $\left.\Gamma_{i}=\left(1-\beta_{i}^{2}\right)^{-1 / 2}\right)$, into an ambient medium of constant density $n=1 n_{0} \mathrm{~cm}^{-3}$. The ejecta transfer their energy to the ambient medium at the characteristic radius $\left(R_{\mathrm{dec}}\right)$ at which point they have swept up a mass comparable to $\sim 1 / \Gamma_{i}$ of their rest mass:

$$
\begin{aligned}
R_{\mathrm{dec}} & \approx\left(\frac{3 E_{K}}{4 \pi n m_{p} c^{2} \Gamma_{i}^{2} \beta_{i}^{2}}\right)^{1 / 3} \\
& \approx 6 \times 10^{17} \mathrm{~cm} E_{K, 51}^{1 / 3} n_{0}^{-1 / 3} \Gamma_{i}^{-2 / 3} \beta_{i}^{-2 / 3} .
\end{aligned}
$$

This occurs at the deceleration timescale:

$$
t_{\mathrm{dec}} \approx R_{\mathrm{dec}} / 2 c \beta_{i} \Gamma_{i}^{2} \approx 115 \mathrm{~d} E_{K, 51}^{1 / 3} n_{0}^{-1 / 3} \beta_{i}^{-5 / 3} \Gamma_{i}^{-8 / 3} .
$$

Prior to $t_{\mathrm{dec}}$ the radio brightness will rise, so this timescale defines a minimum peak time for radio transients (although the peak time could be longer; see below).

If the observing frequency $\left(\nu_{\mathrm{obs}}=1 \nu_{\mathrm{GHz}} \mathrm{GHz}\right)$ is located above both the synchrotron peak frequency $\left(\nu_{m}\right)$ and the self-absorption frequency $\left(\nu_{a}\right)$, then the peak brightness is achieved at $t_{\mathrm{dec}}$, and is given by (Nakar \& Piran 2011):

$$
F_{\nu, \mathrm{dec}} \approx 0.05 \text { mJy } E_{K, 51} n_{0}^{0.83} \epsilon_{e,-1}^{1.3} \epsilon_{B,-2}^{0.83} \beta_{i}^{2.3} D_{L, 28}^{-2} \nu_{\mathrm{GHz}}^{-0.65} .
$$

Here $D_{L}=10^{28} D_{L, 28} \mathrm{~cm}$ is the luminosity distance. We also make the standard assumption that electrons are accelerated at the shock front into a power-law energy distribution $N(E) \propto E^{-p}$ with $p=2.3$ above a minimum Lorentz factor of $\gamma_{m}$, and that $\epsilon_{B}=0.01 \epsilon_{B,-2}$ and $\epsilon_{e}=$ $0.1 \epsilon_{e,-1}$ are the fractions of post-shock energy in the magnetic field and relativistic electrons, respectively.

Some of the transients we consider in this work (e.g., SNe, binary neutron star mergers) produce only nonor mildly-relativistic ejecta and are essentially spherical with isotropic emission at all times. Other transients (e.g. GRBs, jetted TDEs) produce ultra-relativistic ejects in tightly collimated jets ${ }^{3}$ with opening angles of $\theta_{j} \ll 1$. However, when viewed in an initial off-axis direction, the emission from these sources can also be approximated as being spherically symmetric once the shocked matter decelerates to sub-relativistic velocities and spreads laterally into the observer's line of sight (e.g., Zhang \& MacFadyen 2009; Wygoda et al. 2011). At this

${ }^{3}$ GRB jets have $\Gamma_{i} \gtrsim 100$ and $\theta_{j} \sim 0.1$ (e.g., Frail et al. 2001), while TDE jets have $\Gamma_{i} \sim 10$ and $\theta_{j} \sim 0.1$ (e.g., Metzger et al. 2012; Berger et al. 2012). 
point the radio emission is no longer strongly beamed, and $t_{\mathrm{dec}}$ and $F_{\nu \text {,dec }}$ can be approximated using $\beta_{i} \approx 1$ (Nakar \& Piran 2011).

From Equations 2 and 3 it is clear that the luminosities of synchrotron transients scale linearly in proportion to their kinetic energy, but that more energetic events also evolve on a slower characteristic timescale, $t_{\mathrm{dec}} \propto E_{K}^{1 / 3}$. The timescale can be further increased by cosmological time dilation, $t_{\mathrm{dec}} \propto(1+z)$, if such events are energetic enough to be detectable at substantial redshifts.

Explosions in high density environments are also more luminous (Equation 3) but exhibit shorter timescales $\left(t_{\text {dec }} \propto n^{-1 / 3}\right)$. High densities could thus in principle lead to very luminous short-duration transients. However, as the density increases synchrotron self-absorption becomes important and eventually suppresses the brightness and leads to a longer duration because the relevant timescale to reach peak brightness is no longer $t_{\mathrm{dec}}$ but instead the timescale at which $\nu_{a}=\nu_{\text {obs }}$, i.e., when the optical depth is of order unity. The self-absorption frequency at $t_{\mathrm{dec}}$ is given by (Nakar \& Piran 2011):

$$
\nu_{a}\left(t_{\mathrm{dec}}\right) \approx 0.8 \mathrm{GHz} E_{K, 51}^{0.11} n_{0}^{0.49} \epsilon_{B,-2}^{0.34} \epsilon_{e,-1}^{0.41} \beta_{i}^{1.3} .
$$

Thus, self-absorption may play a significant role in suppressing the brightness and extending the timescale of transients, especially at sub- $\mathrm{GHz}$ frequencies. On the other hand, the steep self-absorbed spectrum $\left(F_{\nu} \propto \nu^{2}\right.$ or $\nu^{5 / 2}$ ) can lead to a positive $\mathrm{K}$-correction for sources that are energetic enough to be detectable at cosmological distances (e.g., on-axis GRB afterglows; Frail et al. 2006).

Finally, we note that $t_{\mathrm{dec}}$ can be very short for relativistic sources $\left(t_{\mathrm{dec}} \propto \Gamma_{i}^{-8 / 3}\right)$ so in principle such sources can exhibit short timescales. However, for relativistic sources the relevant peak timescale at radio frequencies is not determined by $t_{\mathrm{dec}}$ because on this timescale the synchrotron peak frequency generically obeys $\nu_{m} \gtrsim \nu_{\text {obs }}$. The value of $\nu_{m}$ is determined by the Lorentz factor of the minimum energy electrons, $\gamma_{m} \approx 40 \epsilon_{e,-1} \Gamma$ (Sari et al. 1998), such that for large values of $\Gamma$ it is located above $\mathrm{GHz}$ frequencies until the outflow decelerates significantly. Thus, the radio light curve at $\nu_{\text {obs }}$ will reach peak brightness only when $\nu_{m}=\nu_{\text {obs }}$ (as long as $\nu_{a} \lesssim \nu_{m}$; otherwise the peak will occur when $\left.\nu_{a}=\nu_{\mathrm{obs}}\right)$. The value of $\nu_{m}$ for relativistic sources in a constant density medium (for $\nu_{m} \gtrsim \nu_{a}$ ) is given by (Granot \& Sari 2002):

$$
\nu_{m} \approx 1.0 \mathrm{GHz} E_{\mathrm{K}, \text { iso }, 53}^{1 / 2} \epsilon_{e,-1}^{2} \epsilon_{B,-2}^{1 / 2} t_{2}^{-3 / 2},
$$

where $E_{\mathrm{K} \text {,iso }}$ is the isotropic-equivalent energy, which takes into account the collimation of relativistic sources, the scaling to $10^{53} \mathrm{erg}$ is used since a typical beaming correction of $\sim 0.01$ will give a fiducial $E_{K} \approx 10^{51} \mathrm{erg}$, and $t=100 t_{2} \mathrm{~d}$ is the time since explosion. Thus, relativistic transients with $\Gamma_{i} \gg 1$ have a timescale for peak emission at radio frequencies that is much longer than $t_{\text {dec }}$.

To summarize, for non-relativistic transients, or initially relativistic off-axis transients, the characteristic timescale at radio frequencies is generally determined by $t_{\mathrm{dec}}$. However, if the density is sufficiently high, such that $\nu_{a}\left(t_{\mathrm{dec}}\right) \gtrsim \nu_{\mathrm{obs}}$, the peak time will be longer, defined by time at which $\nu_{a}=\nu_{\mathrm{obs}}$. Similarly, if the source is relativistic such that $\nu_{m}\left(t_{\mathrm{dec}}\right) \gtrsim \nu_{\mathrm{obs}}$ the peak time will also be longer than $t_{\mathrm{dec}}$, defined by the time at which $\nu_{m}=\nu_{\mathrm{obs}}$. This means that for synchrotron transients the timescale at a given luminosity cannot be made arbitrarily short. The boundary defining the allowed phasespace of luminosity and timescale based on the above considerations is shown in Figure 1. This boundary is based on an initially relativistic source decelerating into a constant density ambient medium, and with $\nu_{a} \lesssim \nu_{m}$. For any other case (e.g., non-relativistic source, $\nu_{a} \gtrsim \nu_{m}$ ) the timescale at a given luminosity will be even longer.

Independent of the generic arguments above, synchrotron sources also obey a basic physical constraint that restricts how rapidly they can exhibit significant brightness variations, namely their brightness temperature $^{4}$ is $T_{B} \lesssim 10^{12} \mathrm{~K}$ (the so-called inverse Compton catastrophe limit; Kellermann \& Pauliny-Toth 1969). The co-moving brightness temperature of an expanding source at a time $t$ is given by (e.g., Kulkarni et al. 1998)

$$
T_{B}^{\prime}=\frac{L_{\nu}}{8 \pi^{2} k} \frac{\Gamma^{2}}{\mathcal{D} \beta^{2} \nu^{2} t^{2}} \lesssim 10^{12} \mathrm{~K},
$$

where $\mathcal{D} \approx \Gamma$ is the Doppler factor in cases when the source is expanding towards the observer. A source cannot vary on a timescale shorter than its light crossing time, $\delta t_{\min } \approx t \beta / 2 \Gamma^{2}$, where the $\sim 1 / 2 \Gamma^{2}$ factor corrects for photon arrival delay in the case of relativistic expansion. Equation 6 can thus be recast as a constrained relation between the timescale and luminosity:

$$
\delta t_{\min }=\left[\frac{L_{\nu}}{16 \pi^{2} k T_{\mathrm{B}}^{\prime} \nu^{2} \Gamma^{3}}\right]^{1 / 2} \approx 80 \mathrm{~d} \Gamma^{-3 / 2} L_{\nu, 30}^{1 / 2} \nu_{\mathrm{GHz}}^{-1}
$$

where $L_{\nu, 30} \equiv L_{\nu} /\left(10^{30} \mathrm{erg} \mathrm{s}^{-1} \mathrm{~Hz}^{-1}\right)$ and the righthand term takes $T_{\mathrm{B}}^{\prime}=10^{12} \mathrm{~K}$. This leads to the same conclusion that more luminous sources have longer characteristic timescales. We show the boundary based on the condition $T_{B} \lesssim 10^{12} \mathrm{~K}$ for sources with $\Gamma \beta=1$ in Figure 1. This boundary is remarkably similar to the one defined by the synchrotron model considerations discussed above.

Both considerations imply that mJy-level GHz transients, which are roughly at the detection threshold of planned near-term surveys (Table 2), will have characteristic durations of $\gtrsim 100 \mathrm{~d}$ at cosmological distances. Shorter durations are possible, but only if the luminosities are correspondingly lower, making the detection volume much smaller for such sources; i.e., they will only be detectable if they have a high volumetric rate. For a fixed transient luminosity, timescale, and volumetric rate, it is straight-forward to compute the instantaneous number of events on the sky above a given flux density threshold $\left(F_{\nu, \lim }\right)$ :

$$
N_{\text {char }}=\frac{4 \pi}{3}\left(\frac{L_{\nu}}{4 \pi F_{\nu, \text { lim }}}\right)^{3 / 2} \mathcal{R} t_{\text {dur }} \approx 0.9 L_{\nu, 30}^{3 / 2} \mathcal{R} t_{\text {dur }, 2},(8)
$$

where $\mathcal{R}$ is in units of $\mathrm{Gpc}^{-3} \mathrm{yr}^{-1}$ and we use $F_{\nu, \lim }=1$ mJy. In Figure 1 we show contours of $\mathcal{R}$ such that one

${ }^{4}$ We note that the variability timescales and brightness temperatures for various radio sources were recently compiled by Pietka et al. (2015). 


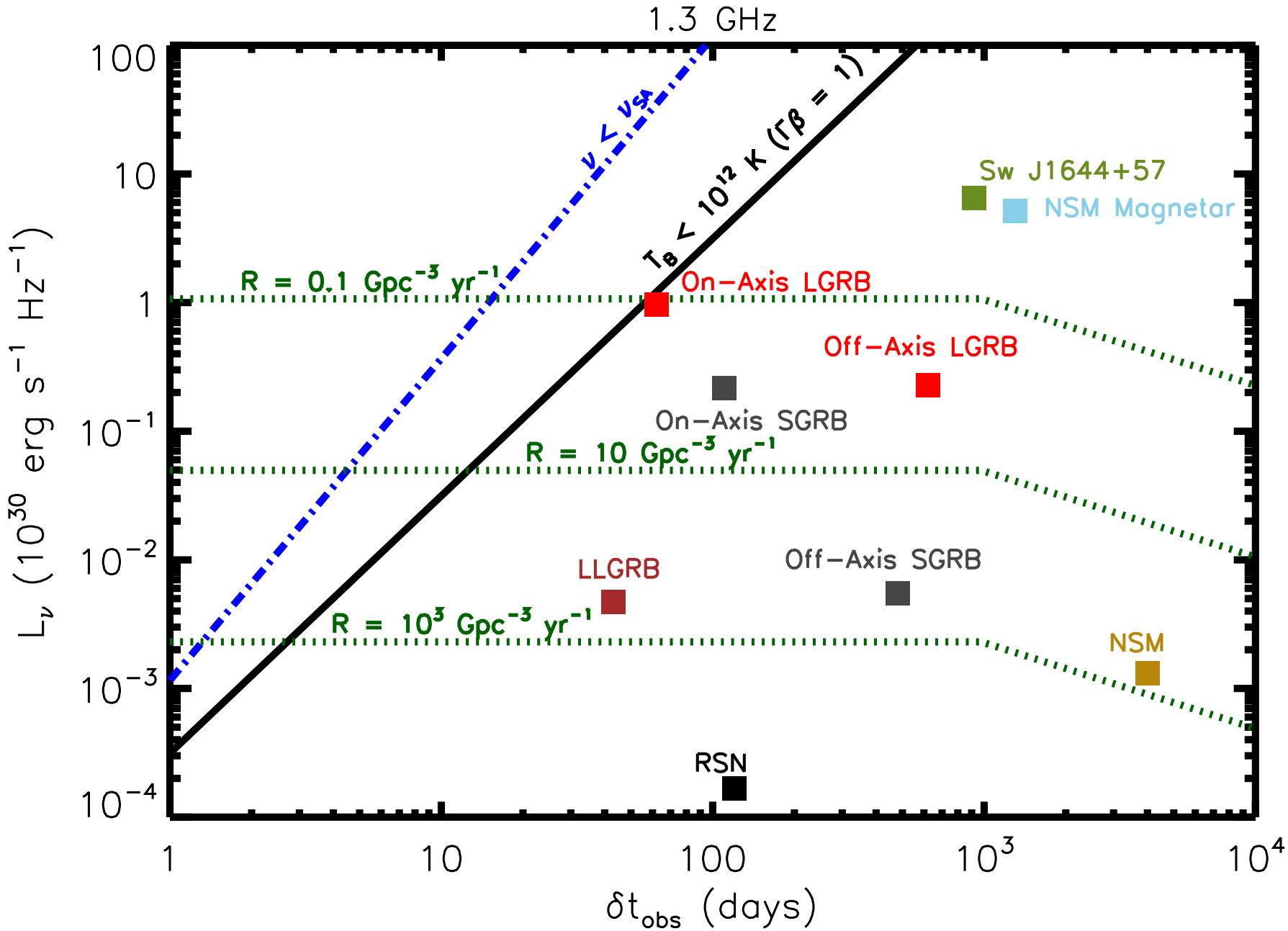

FIG. 1.- Phase-space of peak luminosity and timescale for transient synchrotron sources at a fiducial frequency of 1.3 GHz. Sources in the region of phase-space above the solid black line will violate the brightness temperature constraint $T_{B} \lesssim 10^{12} \mathrm{~K}$ (Equation 7 ). An independent constraint on the allowed phase space is given by the condition that $\nu_{m}, \nu_{a}<\nu_{\mathrm{obs}}$ (Section 2.1; dot-dashed blue line). The green dotted lines indicate the minimum volumetric rate of transients required for at least possible one detection across the entire sky for a three-year survey with a limiting sensitivity of $1 \mathrm{mJy}(\S 2.1)$. Transients with lower peak luminosities (and hence potentially shorter peak timescales) can only be detected within smaller volumes and therefore require higher intrinsic rates. These minimum rates can be compared to actual transient rates as summarized in Table 1. Finally, colored squares denote the peak luminosity and timescale of the various transients considered in this paper: on- and off-axis LGRBs (red), on- and off-axis short SGRBs (gray), neutron star binary mergers with prompt black hole formation (NSM: tan), neutron star binary mergers with a magnetar remnant (NSM magnetar: blue), on-axis jetted TDEs (Sw J1644+57: light green), low luminosity GRB (LLGRB: brown), and Type Ib/c SNe (RSN: black). We note that all of these sources indeed obey the basic luminosity-timescale limits discussed in $\S 2.1$.

event could possibly be detected in a three-year transient survey: given values for $L_{\nu}$ and $t_{\text {dur }}$ we find $N_{\text {char }}(\mathcal{R})=1$ in Equation 8, adopting $t_{\mathrm{dur}}=3 \mathrm{yr}$ if the actual transient duration is shorter than this value. We find that even with this generous definition of detectability, transients varying on a timescale as short as $\sim 3$ days are only detectable if their volumetric rate is $\gtrsim 10^{3} \mathrm{Gpc}^{-3} \mathrm{yr}^{-1}$. This is much higher than the rate of all known extragalactic transients with the exception of Type Ib/c SNe (although the latter are non-relativistic sources and thus vary on much longer timescales than indicated by the condition $\left.T_{B} \approx 10^{12} \mathrm{~K}\right)$. For more reasonable volumetric rates of relativistic transients $\left(\lesssim 1 \mathrm{Gpc}^{-3} \mathrm{yr}^{-1}\right.$; Table 1$)$ the minimum timescale is $\sim 30$ days; this timescale increases to $\sim 100$ days for a detection rate of a few events per year. Thus, extragalactic synchrotron radio transients with timescales of $\ll 100$ days are unlikely to be detected in upcoming surveys.

\subsection{Models and Volumetric Rates}

In Figure 2 we show the light curves for the various transients that we consider in this paper at four frequencies $(0.15,1.3,3$, and $150 \mathrm{GHz})$ characteristic of planned and hypothetical surveys (Table 2 and §3). As can be seen in Figure 1, these transients span a wide range in the luminosity-timescale phase-space and therefore provide a broad view of extragalactic transients. It is also clear from Figure 1 that these transients indeed obey the boundaries defined by the basic synchrotron model consideration discussed above and by the condition $T_{B} \lesssim 10^{12} \mathrm{~K}$. Finally, by comparing the actual volumetric rates of these transients (Table 1 ) to the contours showing the rates required for our fiducial level of observability (one event brighter than $\sim 1$ mJy on the sky over three years), it is already clear that few detections will be made in $\mathrm{GHz}$ surveys with mJy sensitivity.

In the following sections we discuss each class of tran- 
sients in detail. Unless otherwise noted we adopt the microphysical shock parameters $\epsilon_{e}=0.1, \epsilon_{B}=0.01$, and $p=2.3$, typical of those found or used for known extragalactic transients.

\subsubsection{Classical Long GRBs}

The afterglows of long GRBs (LGRBs) are powered by the deceleration of an ultra-relativistic jet by interaction with the circumburst medium (Mészáros \& Rees 1997). The isotropic equivalent energies of classical LGRBs are large, $E_{\text {iso }} \approx 10^{53}-10^{54} \mathrm{erg}$, but with typical jet opening angles of $\theta_{j} \sim 0.1$ (Bloom et al. 2003) the beamingcorrected energies are $\sim 10^{50}-10^{52}$ erg (e.g., Frail et al. 2001; Berger et al. 2003; Cenko et al. 2012). This has been confirmed by late-time radio calorimetry (Frail et al. 2000; Berger et al. 2004; Frail et al. 2005; Shivvers \& Berger 2011). LGRB jets interact with either the interstellar medium or the wind of the massive progenitor star, with typical values of $n \sim 0.1-10 \mathrm{~cm}^{-3}$.

Here we consider synchrotron emission from the forward shock, neglecting emission from the reverse shock which travels into the ejecta at early time (e.g., Sari \& Piran 1999). This is justified by the fact that the reverse shock emission is generally fainter than the forward shock emission at the relevant frequencies of $\lesssim \mathrm{GHz}$ (e.g., Kulkarni et al. 1999; Laskar et al. 2013). However, at the high frequencies relevant to millimeter surveys, the reverse shock emission can be substantially brighter than the forward shock for $\sim$ day (Laskar et al. 2013); we discuss the implications of this point in $\S 6$.

The observed light curves of LGRB radio afterglows also depend on the observer viewing angle with respect to the jet axis, $\theta_{\text {obs }}$. We thus consider both on- and offaxis LGRB afterglow light curves, based on the relativistic numerical hydrodynamical simulations of van Eerten et al. $(2010)^{5}$, including self-absorption. We utilize a model with properties typical of observed LGRBs: $\theta_{j}=$ $\theta_{\text {obs }}=0.2, E_{K}=10^{51} \mathrm{erg}, n=1 \mathrm{~cm}^{-3}$, and $p=2.5$, as well as off-axis ("orphan") jets with $\theta_{\text {obs }}=0.4,0.8,1.57$ (Figure 2). On-axis events, though more luminous, represent only a small fraction, $f_{\text {obs }}=\int_{\theta_{1}}^{\theta_{2}} \sin \theta d \theta$, of the total event rate; the integration from $\theta_{1}=\theta_{\text {obs }} / 2$ to $\theta_{2}=\theta_{\text {obs }}$ covers the approximate range of solid angle covered by observers with viewing angle $\theta \approx \theta_{\text {obs }}$. Depending on observing frequency, depth, and cadence of the survey, detection rates may be dominated by on- or off-axis events.

For the local volumetric rate of on-axis LGRBs we adopt a fiducial value of $\mathcal{R}_{\mathrm{LGRB}}\left(\theta_{\mathrm{obs}} \lesssim \theta_{\mathrm{j}}\right)=0.3$ $\mathrm{Gpc}^{-3} \mathrm{yr}^{-1}$, motivated as follows. Wanderman \& Piran (2010) estimate $\mathcal{R}_{\mathrm{LGRB}} \sim 1 \mathrm{Gpc}^{-3} \mathrm{yr}^{-1}$ for events with isotropic luminosity of $L_{\text {iso }} \gtrsim 10^{50} \mathrm{erg} \mathrm{s}^{-1}\left(E_{\text {iso }} \sim 10^{51}\right.$ erg for a typical LGRB duration of 10 seconds). The LGRB luminosity function measured by Swift is approximately flat per unit $\log L_{\text {iso }}$ below the characteristic value $L_{\text {iso }} \approx 10^{52} \mathrm{erg} \mathrm{s}^{-1}$ (corresponding to the same isotropic energy $\sim 10^{53}$ erg in our assumed afterglow calculation above). Thus the rate of events with $E_{\text {iso }} \sim 10^{53} \mathrm{erg}$ is $\sim 1 / 3$ of the rate of events with $E_{\text {iso }} \gtrsim 10^{51} \mathrm{ergs}$, or $\approx 0.3$ $\mathrm{Gpc}^{-3} \mathrm{yr}^{-1}$. For off-axis LGRBs we scale the on-axis

\footnotetext{
${ }^{5}$ http://cosmo.nyu.edu/afterglowlibrary
}

rate according to $\mathcal{R}_{\mathrm{LGRB}}\left(\theta_{\mathrm{obs}}\right)=\mathcal{R}_{\mathrm{LGRB}}\left(\theta_{\mathrm{obs}}=\theta_{\mathrm{j}}\right) f_{\text {obs }}^{-1}$. Finally, the rate is assumed to evolve with redshift in the same manner as the cosmic star formation rate density, according to the model of Cucciati et al. (2012). Although this may not hold strictly (e.g., Yüksel et al. 2008), the uncertainty introduced by this assumption is small compared to that from other approximations.

\subsubsection{Low-Luminosity Long GRBs}

A class of low-luminosity LGRBs (LLGRBs) with lower total beaming-corrected energies of $E_{K} \sim 10^{49} \mathrm{erg}$ and only mildly-relativistic ejecta $\left(\Gamma_{i} \approx 2\right)$ has been identified in recent years (Kulkarni et al. 1998; Soderberg et al. 2004). LLGRBs appear to be roughly isotropic, and it has been suggested that they result from relativistic shock break-out following the core-collapse of massive stars with extended envelopes (e.g., Matzner \& McKee 1999, Nakar \& Sari 2012). We model the radio emission from LLGRBs using the model of Barniol Duran et al. (2014) as fit to the radio afterglow of GRB 980425 (Kulkarni et al. 1998).

We use an LLGRB volumetric rate that is 10 times higher than the beaming-corrected LGRB rate (e.g., Soderberg et al. 2004; Coward 2005; Guetta \& Della Valle 2007). Adopting a total on-axis LGRB rate of $\approx 1$ $\mathrm{Gpc}^{-3} \mathrm{yr}^{-1}$ and a classical LGRB beaming fraction of $\approx 50$ we thus take the volumetric rate of LLGRBs to be $\mathcal{R}_{\mathrm{LLGRB}} \approx 500 \mathrm{Gpc}^{-3} \mathrm{yr}^{-1}$. We note that given their lower energy scale, LLGRBs are only detectable nearby, and therefore redshift evolution of the rate is unimportant.

\subsubsection{Short GRBs}

Short-duration gamma-ray bursts (SGRBs) are also accompanied by afterglow emission, but with beamingcorrected energies of $E_{K} \sim 10^{49}-10^{50} \mathrm{erg}$, lower than in the case of LGRBs (Berger 2014). The circumburst densities are also theoretically expected and observationally inferred to be lower than in the case of LGRBs, with $n \sim 0.001-0.1 \mathrm{~cm}^{-3}$ (e.g., Fong \& Berger 2013). We therefore adopt the low energy afterglow model of van Eerten \& MacFadyen (2011) with $E_{K}=10^{50} \mathrm{erg}$, $n=10^{-3} \mathrm{~cm}^{-1}, \theta_{j}=0.2, \epsilon_{e}=\epsilon_{B}=0.1$, and $p=2.5$. These parameters are consistent with the observed onaxis optical afterglows of SGRBs (Metzger \& Berger $2012)$. In addition to the on-axis case $\left(\theta_{\mathrm{obs}}=0.2\right)$ we also consider off-axis events with $\theta_{\text {obs }}=0.4,0.8,1.57$.

We use a local volumetric rate of on-axis SGRBs of $\mathcal{R}_{\mathrm{SGRB}}=5 \mathrm{Gpc}^{-3} \mathrm{yr}^{-1}$ (e.g., Wanderman \& Piran 2014), and scale it appropriately for off-axis angles as described in $\S 2.2 .1$. As in the case of LLGRBs, redshift evolution of the rate is unimportant due to the low luminosity of these events.

\subsubsection{NS-NS Mergers with Prompt Black Hole Formation}

The merger of two neutron stars, or of a neutron star and a low mass black hole, results in the ejection of a small quantity of mass, $M_{\mathrm{ej}} \sim 10^{-3}-10^{-2} M_{\odot}$, with mildly relativistic velocities, $\beta_{i}=0.1-0.3$ (e.g., Rosswog et al. 2013; Hotokezaka et al. 2013). The interaction of the ejecta with the surrounding interstellar medium will result in synchrotron radio emission similar to that of a supernova remnant (Nakar \& Piran 2011; Hotokezaka \& 

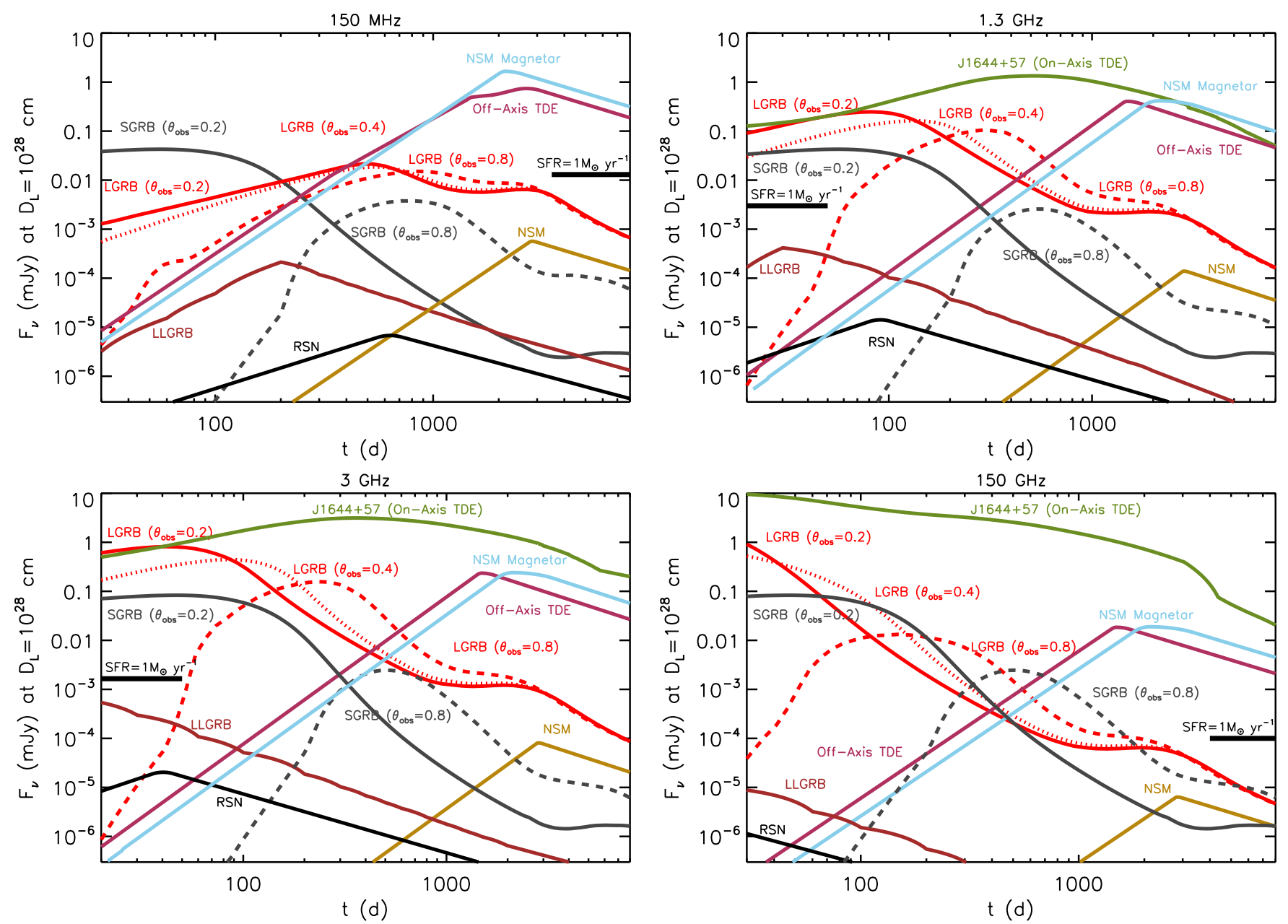

FIG. 2.- Light curves of the various transients considered in this paper (Table 1) at observing frequencies of $150 \mathrm{MHz}$ (upper left), 1.3 $\mathrm{GHz}$ (upper right), $3 \mathrm{GHz}$ (lower left), and $150 \mathrm{GHz}$ (lower right). Sources considered here include: on- and off-axis long GRB afterglows for various observing angles $\theta_{\text {obs }}$ (LGRB: red); low-luminosity LGRB afterglows (LLGRB; brown); Type Ib/c SNe (RSN; black); on- and off-axis short GRB afterglows (SGRB: charcoal); on-axis jetted TDEs (Sw J1644+57: green); off-axis TDEs (maroon); neutron star binary mergers with prompt black hole formation (NSM; tan) and a magnetar remnant (NSM magnetar; blue). Flux densities are normalized to $D_{\mathrm{L}}=10^{28} \mathrm{~cm}(z \approx 0.55)$. In each panel we also annotate the typical flux density of a host galaxy forming stars at a fiducial rate of $1 \mathrm{M} \odot \mathrm{yr}^{-1}$ (horizontal black bars). See Section 2 for further details.

Piran 2015). We calculate the radio emission using the spherical model of Nakar \& Piran (2011) for $\beta_{i}=0.2$, $E_{K}=3 \times 10^{50} \mathrm{erg}\left(M_{\mathrm{ej}}=10^{-2} M_{\odot}\right)$, and $n=0.1 \mathrm{~cm}^{-3}$.

We use a local neutron star merger (NSM) volumetric rate of $\mathcal{R}_{\mathrm{NSM}}=500 \mathrm{Gpc}^{-3} \mathrm{yr}^{-1}$, consistent with that inferred from the Galactic binary pulsar population (e.g., Kim et al. 2013).

\subsubsection{NS-NS Mergers Leaving a Stable NS Remnant 83 White Dwarf Accretion-Induced Collapse}

In some cases neutron star binary mergers may result in the formation of a long-lived neutron star instead of immediate collapse to a black hole (e.g., Metzger et al. 2008; Siegel et al. 2014). This stable remnant is formed rapidly rotating, with a period of $P \sim 1$ ms, and correspondingly large rotational kinetic energy of $E_{\text {rot }} \approx 4 \pi^{2} I / P^{2} \approx 3 \times 10^{52} \mathrm{erg}$, where $I \sim 10^{45} \mathrm{~g} \mathrm{~cm}^{2}$ is the NS moment of inertia. If the merger remnant also possesses a moderately strong dipole magnetic field of $B \gtrsim 10^{13} \mathrm{G}$, then its electromagnetic dipole spin-down will transfer the rotational energy to the small quantity of ejecta, accelerating it to trans-relativistic speeds,
$\Gamma_{i} \simeq E_{\mathrm{rot}} / M_{\mathrm{ej}} c^{2} \gtrsim 1$, before the ejecta have been decelerated by the ISM (Metzger \& Bower 2014). A millisecond neutron star with similar properties may also be formed by the accretion-induced collapse (AIC) of a rotating white dwarf (Usov 1992).

We model the radio emission from both neutron star mergers leaving a stable remnant and AIC events using the Nakar \& Piran (2011) spherical blast wave model ${ }^{6}$ with $\beta_{i}=1, E_{K}=3 \times 10^{52} \mathrm{erg}$, and $n=0.1 \mathrm{~cm}^{-3}$. The energy and velocity of the ejecta are uncertain because they depend, among other things, on how efficiently the NS outflow couples its energy to the merger ejecta (e.g., Bucciantini et al. 2012) and whether a fraction of the NS rotational energy is instead lost to gravitational radiation (e.g., Corsi \& Mészáros 2009). The energy will also be smaller if the NS collapses to a black hole before the bulk of its rotational energy is extracted; however,

${ }^{6}$ Piro \& Kulkarni (2013) argue that the nascent pulsar wind nebula created by AIC will also produce a radio transient, but we estimate that the synchrotron radiation from the ISM interaction will dominate the nebular emission unless the ISM density is exceedingly low, $n \ll 10^{-4} \epsilon_{e,-1}^{-1} \mathrm{~cm}^{-3}$. 
the much brighter radio emission from stable NS remnants imply that they will dominate the radio detection rate if such objects form at all. A higher ISM density is expected compared to the case of prompt $\mathrm{BH}$ formation because mergers leaving stable remnants may occur preferentially within their host galaxies due to the small natal kicks that are expected to accompany the birth of the lowest mass neutron stars formed in electron capture supernovae (e.g., Belczynski et al. 2008).

The rate of mergers leaving stable NS remnants is unknown, as it depends on the equation of state of ultrahigh density matter and on the mass distribution of binary neutron stars. We therefore scale the rate to $1 \%$ of the total NSM rate, i.e., $\sim 5 \mathrm{Gpc}^{-3} \mathrm{yr}^{-1}$; as we show later on, much higher volumetric rates may already be ruled out by past radio surveys due to the extremely high luminosities of these events (see in particular the FIRST/NVSS constraints from Levinson et al. (2002) in Figure 3$)$. The rate of AIC is also uncertain, with population synthesis estimates spanning $\mathcal{R}_{\text {AIC }} \sim 10-10^{3}$ $\mathrm{Gpc}^{-3} \mathrm{yr}^{-1}$ (Yungelson \& Livio 1998), and the fraction of WDs that are rapidly rotating at the time of collapse is even less certain. We scale the volumetric rates with redshift according to the cosmic star formation rate density (§2.2.1), assuming that the characteristic delay time between star formation and these events is relatively short, $\ll$ Gyr (e.g., Leibler \& Berger 2010). We note that if the typical delays are in fact much longer, the observed rate will be suppressed due to the loss of events at $z \gtrsim 1$. Given that both NS mergers with stable magnetar remnants and rotating AIC events are predicted to produce similar radio transients and that the rates of both are highly uncertain, for notational simplicity we hereafter denote both models as "NSM-magnetar."

\subsubsection{On-Axis Jetted Tidal Disruption Event $(S w J 1644+57)$}

The transient event Swift J164449.3 + 573451 (hereafter Sw J1644+57) exhibited several properties that led to its interpretation as a tidal disruption event with a relativistic jet (Bloom et al. 2011; Burrows et al. 2011; Levan et al. 2011; Zauderer et al. 2011). The relativistic outflow produced long-lived radio synchrotron emission, which is still detected at the present (Giannios \& Metzger 2011; Zauderer et al. 2011; Berger et al. 2012; Metzger et al. 2012; Zauderer et al. 2013). A second TDE candidate with similar X-ray and radio emission, Sw J2058+05, has been reported by Cenko et al. (2012).

We model the radio emission from on-axis jetted TDEs at $\gtrsim 1 \mathrm{GHz}$ directly using the radio light curves of Sw J1644+57 as a template (Zauderer et al. 2011; Berger et al. 2012; Zauderer et al. 2013). Since data are not yet available at $150 \mathrm{MHz}$ (Cendes et al. 2014), we assume that the on-axis emission at this frequency is identical to the isotropic, off-axis model (see below). This is justified by the high self-absorption frequency at early times, which will strongly suppress the low frequency emission for on-axis sources.

We estimate the local volumetric rate of on-axis jetted TDEs in two ways. First, the fact that one event was detected by Swift in $\Delta t \approx 10$ years of monitoring to a redshift $z=0.35$ (co-moving volume of $V \approx 11 \mathrm{Gpc}^{-3}$ ) suggests a rate of $\mathcal{R}_{\mathrm{SwJ} 1644+57} \sim 1 /(V \Delta T) \sim 0.01 \mathrm{Gpc}^{-3}$ $\mathrm{yr}^{-1}$. Alternatively, we can utilize the theoretically expected TDE rate of $\sim 10^{-5}-10^{-4}$ galaxy ${ }^{-1} \mathrm{yr}^{-1}$ (e.g., Wang \& Merritt 2004; Stone \& Metzger 2014), which given a local galaxy density of $\sim 10^{-2} \mathrm{Mpc}^{-3}$ corresponds to a TDE rate of $\sim 100-1000 \mathrm{Gpc}^{-3} \mathrm{yr}^{-1}$. Assuming a beaming correction of $\sim 100$ for Sw J1644+57 (Zauderer et al. 2011; Berger et al. 2012; Metzger et al. 2012 ) and that $\lesssim 10 \%$ of TDEs produce relativistic jets (Bower et al. 2013; van Velzen et al. 2013), this corresponds to a volumetric rate of $\lesssim 0.1-1 \mathrm{Gpc}^{-3} \mathrm{yr}^{-1}$ for on-axis jetted TDEs, consistent with the empirical estimate. In what follows we adopt the conservative onaxis rate of $\mathcal{R}_{\mathrm{SwJ} 1644+57} \sim 0.01 \mathrm{Gpc}^{-3} \mathrm{yr}^{-1}$ but note that the true local rate may be higher by at least an order of magnitude, especially when considering that the conditions to produce a mildly-relativistic outflow with $\Gamma_{i} \gtrsim 2$, which will produce radio emission, may be less stringent than those required to produce bright X-ray emission (and hence that Sw J1644+57 provides only a lower limit on the rate).

We scale the TDE rate with redshift using the volume density of $\sim 10^{5}-10^{7} M_{\odot}$ black holes, for which we employ the model of Sijacki et al. (2014) (their Figure 2). This model predicts that the TDE rate decreases by a factor of $\sim 2$ between $z=0$ and $z \sim 4$, before decreasing more rapidly at $z \gtrsim 4$ (although see Hopkins et al. 2007 who find a more rapid evolution with redshift). Beyond the uncertainties in the evolution of the supermassive black hole volume density, the TDE rate per galaxy also depends on other factors, such as the mass distribution of stars in galactic nuclei, which could also evolve strongly with redshift. Our assumed rate evolution thus represents a best guess given the lack of detailed studies.

\subsubsection{Off-Axis Jetted Tidal Disruption Events}

For Sw J1644+57 the initial jet Lorentz factor was estimated to be $\Gamma_{i} \sim 10$ (Metzger et al. 2012; Berger et al. 2012 ), corresponding to a beaming fraction $f_{\mathrm{b}} \sim 0.01$. Off-axis jetted TDEs may thus dominate on-axis events at radio frequencies (Giannios \& Metzger 2011; van Velzen et al. 2011; Donnarumma et al. 2015). Detailed predictions for the off-axis radio light curves of jetted TDEs are not currently available, but because the emission is expected to be relatively isotropic and only mildlyrelativistic at late times (e.g., Berger et al. 2012), we assume a spherical blast wave model. We utilize the Nakar \& Piran (2011) model with $\beta_{i}=1, E_{K}=10^{52}$ erg, $n=0.1 \mathrm{~cm}^{-3}, \epsilon_{e}=0.2, \epsilon_{\mathrm{B}}=0.01$, and $p=2.3$, chosen to produce a $1.4 \mathrm{GHz}$ peak flux density in agreement with that of Sw J1644+57 (since at late times the jet has spread laterally and its emission is approximately isotropic).

We assume the same evolution of the volumetric rate with redshift as in the on-axis case, but with a local volumetric rate of $\sim 1 \mathrm{Gpc}^{-3} \mathrm{yr}^{-1}$ which is $f_{\mathrm{b}}^{-1} \sim 100$ times the on-axis rate.

\subsubsection{Type $I b / c$ Supernovae}

Core-collapse supernovae $(\mathrm{SNe})$ produce radio emission as the fastest ejecta interact with the dense gas of the progenitor's stellar wind (e.g., Chevalier 1982). Radio emission is observed in both stripped-envelope Type Ib/c SNe (e.g., Berger et al. 2003) and Type II 
SNe (e.g., Weiler et al. 2002). In fact, the few genuine radio transient discoveries to date have been identified as Type II SNe: a SN in Markarian 297 (Yin \& Heeschen 1991), FIRST J121550.2+130654 (Levinson et al. 2002; Gal-Yam et al. 2006), and SN 2008iz (Brunthaler et al. 2009, 2010). We note, however, that only FIRST J121550.2+130654 was discovered in an untargeted search (using a comparison of NVSS and FIRST with a baseline of about 5 years), while the other two events were discovered in targeted observations of startburst galaxies with elevated core-collapse SN rates. Theory and observations indicate that Type II SNe are actually challenging to discover in the untargeted surveys we consider here. In particular, these sources are dominated by synchrotron self-absorption and free-free absorption such that the most luminous events (generally Type IIn SNe, with $L_{\nu} \sim 10^{28}-10^{29} \mathrm{erg} \mathrm{s}^{-1} \mathrm{~Hz}^{-1}$ ) have typical timescales of about a decade at $\sim 1 \mathrm{GHz}$ (Chevalier 2006), and are thus indistinguishable from steady sources; Type II SNe that vary on timescales of $\sim$ year (generally Type IIP SNe) have much lower luminosities $\left(L_{\nu} \lesssim 10^{27} \mathrm{erg} \mathrm{s}^{-1} \mathrm{~Hz}^{-1}\right)$ and are thus detectable in a negligible volume. This indicates that Type II SNe may appear in the source lists of radio surveys, but will not be easily identified as transients within the time-frame of the surveys.

Here we instead focus on Type Ib/c SNe, for which the radio emission evolves more rapidly due to the presence of faster ejecta $(\beta \sim 0.1-0.3$; Berger et al. 2003; Chevalier \& Fransson 2006). The Type Ib/c SNe detected in the radio exhibit a wide spread in peak luminosity and timescale $\left(L_{\nu, p} \sim 10^{25}-10^{28} \mathrm{erg} \mathrm{s}^{-1} \mathrm{~Hz}^{-1}\right.$ and $t_{p} \sim 10-1000 \mathrm{~d}$ ), following the general pattern in which the most luminous events evolve most slowly. At the present, the radio luminosity function of Type $\mathrm{Ib} / \mathrm{c}$ SNe has not been quantified so here we use data for the well-sampled, and relatively typical, event SN 2008D (Soderberg et al. 2008), modeled using the formulation of Soderberg et al. (2005). We note that the SN model is somewhat different from the models we consider above because the ejecta have a large spread in velocity, with the energy distributed approximately as $E_{K}(>v) \sim v^{-5}$ (Chevalier 1982).

To estimate the local volumetric rate we note that the fraction of Type Ib/c SNe with detectable radio emission approaches unity only for the nearest events $(\lesssim 15 \mathrm{Mpc})$. About $\sim 1 / 4$ of events are detected within the distance of SN 2008D ( $\approx 27 \mathrm{Mpc})$. We therefore assume a fiducial rate of $\mathcal{R}_{\mathrm{Ibc}}=5 \times 10^{3} \mathrm{Gpc}^{-3} \mathrm{yr}^{-1}$, corresponding to one quarter of the Type Ib/c SN volumetric rate (Guetta \& Della Valle 2007).

\section{RADIO SURVEYS}

A variety of time-domain radio surveys covering a broad range of frequencies, sensitivities, cadences, and angular resolutions are slated to come online over the course of the next decade and beyond. This section summarizes the set of surveys that we simulate in this work. Some are currently in advanced stages of development or are even already taking data, while others are still in early stages of planning. We also consider hypothetical surveys, some of which depend on judicious temporal binning of planned high-cadence surveys, motivated by the transient timescale arguments we presented in $\S 2.1$. The key parameters of the various surveys are summarized in Table 2.

\subsection{ASKAP VAST}

The Australian Square Kilometer Array Pathfinder (ASKAP) is a precursor and technology development platform for the SKA, under development in Western Australia. Its wide field of view $\left(\sim 30 \mathrm{deg}^{2}\right)$ and moderately high sensitivity $\left(\sim\right.$ mJy beam ${ }^{-1}$ for a $10 \mathrm{~s}$ integration) will enable fast, sensitive all-sky surveys (Johnston et al. 2008b). The ASKAP Survey for Variables and Slow Transients (VAST; Murphy et al. 2013) comprises several surveys aimed at detecting transients in the $1.1-1.4 \mathrm{GHz}$ frequency range: 'VAST-Wide' will survey $10^{4} \mathrm{deg}^{2}$ per day to a $1 \sigma \mathrm{rms}$ sensitivity of $0.5 \mathrm{mJy}$, while VASTDeep Multi-Field (hereafter 'VAST-Deep'), will survey $10^{4} \mathrm{deg}^{2}$ to $0.05 \mathrm{mJy}(1 \sigma)$ every $\sim$ year. We also consider the planned Deep Single Field survey (hereafter 'VASTDeep-SF') with $30 \mathrm{deg}^{2}$ surveyed daily to 0.05 mJy $(1 \sigma)$.

We assume that both VAST-Wide and VAST-Deep ${ }^{7}$ will last for 3 years, while VAST-Deep-SF is assumed to last for 1 year. Although a dynamical cadence has been proposed for VAST-Deep, we assume a uniform cadence since details of the dynamical cadence have not yet been specified.

For the daily-cadence surveys VAST-Wide and VASTDeep-SF we also consider the possibility of co-adding the daily exposures to create deeper maps with a slower effective cadence, motivated by the long durations of extragalactic synchrotron transient sources. We construct two separate mock surveys ('VAST-Wide-Stack' and 'VASTDeep-SF-Stack') by averaging 30 separate daily observations of VAST-Wide and VAST-Deep-SF to achieve effective sensitivities of $0.09 \mathrm{mJy}$ and $0.01 \mathrm{mJy}$, respectively. We note that the latter is limited to the estimated confusion limit of ASKAP.

\subsection{LOFAR Radio Sky Monitor}

The LOw-Frequency ARrray (LOFAR) operates between about 30 and $240 \mathrm{MHz}$ (van Haarlem et al. 2013). The 32 core stations of LOFAR will ultimately be able to return up to 24 individual beams, which will cover a patch of the sky as a Radio Sky Monitor (RSM; Fender et al. 2006; Best \& LOFAR-UK Consortium 2008; Fender 2012). The currently operational LOFAR RSM Zenith Monitoring Program covers $\approx 1500 \mathrm{deg}^{2}$ at $\approx 150 \mathrm{MHz}$ to a $1 \sigma \mathrm{rms}$ sensitivity of $12 \mathrm{mJy}$ (J. Broderick, private communication). A mean cadence timescale of $\sim 1$ month is planned, corresponding to 36 observing epochs for an assumed three year survey. Beyond the current plan, we also consider a more ambitious hypothetical survey ('LOFAR-Expanded'), which we assume reaches a factor of 10 times deeper and covers a significantly larger sky area $\left(\sim 10^{4} \mathrm{deg}^{2}\right)$. Though ambitious in relation to LOFAR's existing capabilities, such a program is similar to the original RSM.

\subsection{VLA Sky Survey}

\footnotetext{
7 The terms 'Deep' and 'Wide' are somewhat misleading; both surveys cover the same sky area, with the real distinction being the higher cadence and lower per-epoch sensitivity of VAST-Wide. We nevertheless adopt this terminology here to avoid confusion with existing literature.
} 
TABLE 2

Adopted Radio Survey Parameters

\begin{tabular}{lccccccc}
\hline \hline \multicolumn{1}{c}{ Name } & $\begin{array}{c}\theta_{\text {res }} \\
(\operatorname{arcsec})\end{array}$ & $\begin{array}{c}\nu \\
(\mathrm{GHz})\end{array}$ & $\begin{array}{c}\sigma^{(a)} \\
(\mathrm{mJy})\end{array}$ & $\begin{array}{c}\text { Area } \\
\left(\mathrm{deg}^{2}\right)\end{array}$ & $\begin{array}{c}\Delta T^{(b)} \\
(\mathrm{d})\end{array}$ & $\mathrm{N}_{\text {obs }}(c)$ & Ref. \\
\hline LOFAR & 80 & 0.15 & 12 & 1500 & 30 & 36 & 1 \\
LOFAR-Expanded & 80 & 0.15 & 1.2 & $10^{4}$ & 30 & 36 & 1 \\
SKA-Low & 11 & 0.15 & 0.004 & $10^{4}$ & 90 & 13 & 3 \\
\hline VAST-Wide & 10 & 1.3 & 0.5 & $10^{4}$ & 1 & 1096 & 2 \\
VAST-Wide-Stack & 10 & 1.3 & 0.09 & $10^{4}$ & 30 & 36 & 2 \\
VAST-Deep & 10 & 1.3 & 0.05 & $10^{4}$ & 365 & 4 & 2 \\
VAST-Deep-SF & 10 & 1.3 & 0.05 & 30 & 1 & 365 & 2 \\
VAST-Deep-SF-Stack & 10 & 1.3 & 0.01 & 30 & 30 & 36 & 2 \\
SKA & 0.9 & 1.3 & 0.009 & $10^{4}$ & 90 & 13 & 3 \\
SKA-Expanded & 0.9 & 1.3 & 0.006 & $3 \times 10^{4}$ & 30 & 36 & 3 \\
\hline VLASS-Wide & 2 & 3 & 0.1 & $10^{4}$ & 365 & 4 & - \\
VLASS-Deep & 0.6 & 3 & 0.003 & 10 & 365 & 4 & - \\
\hline CMB & 840 & 150 & 1.0 & $10^{4}$ & 1 & 1096 & 4 \\
CMB-Stack-1 & 840 & 150 & 0.31 & $10^{4}$ & 10 & 110 & 4 \\
CMB-Stack-2 & 840 & 150 & 0.1 & $10^{4}$ & 100 & 11 & 4 \\
\hline
\end{tabular}

NoTE. - All surveys are assumed to last 3 years, except for VAST-Deep-SF with a duration of 1 year. ${ }^{(a)}$ RMS $1 \sigma$ sensitivity; ${ }^{(b)}$ Cadence; ${ }^{(c)}$ Number of epochs. References: (1) Fender 2012, J. Broderick (private communication); (2) Murphy et al. 2013; (3) Carilli \& Rawlings 2004; (4) B. Johnson, G. Jones, private communication.

A series of large surveys with the upgraded Karl G. Jansky Very Large Array (Perley et al. 2011), collectively known as the VLA Sky Survey (VLASS), has been recently discussed. Although the parameters of the VLASS components are far from settled, we simulate several large surveys using the most recent information available to us. We consider surveys operating in the S-band $(2-4 \mathrm{GHz})$ using a combination of the high-resolution $\mathrm{A}$ and B configurations. The 'VLASS-Wide' survey covers $10^{4} \mathrm{deg}^{2}$ at an rms sensitivity of $0.1 \mathrm{mJy}(1 \sigma)$ over 4 epochs with a yearly cadence. The 'VLASS-Deep' survey covers a much smaller area of $10 \mathrm{deg}^{2}$ with an annual cadence, achieving a $1 \sigma$ sensitivity of $\sim 3 \mu \mathrm{Jy}$. Although it is planned for each epoch of VLASS-Deep to achieve the target sensitivity by revisiting the survey footprint in 10-30 passes over $\sim 3$ months, we consider only the final deep integrations because the timescale for multiple passes is comparable to that of extragalactic synchrotron transients. Finally, a VLASS 'All-Sky' survey covering about $34,000 \mathrm{deg}^{2}$ with a sensitivity of $0.1 \mathrm{mJy}(1 \sigma)$ is also being discussed, but it will be comprised of only 2 epochs, limiting its utility for detailed time-domain studies. We do not consider this survey here.

\subsection{Millimeter Survey}

In recent years $\mathrm{mm}$ and sub-mm telescopes have been developed to study the cosmic microwave background $(\mathrm{CMB})$, which are sensitive, wide-field, and exceptionally stable. We consider whether these instruments could be employed for transient discovery. We estimate what a hypothetical ground-based mm survey could achieve by extrapolating the parameters of the proposed design for a polarimeter based on lumped-element kinetic inductance detectors (LEKIDs; Araujo et al. 2014). This design specifies an aperture diameter of $50 \mathrm{~cm}$, with an estimated $\sim 3.2$ mJy sensitivity $(1 \sigma)$, and a daily sky coverage of $\sim 10^{4} \operatorname{deg}^{2}$ (G. Jones, B. Johnson, private communication). For the purpose of our study we consider a large 2-m diameter instrument, achieving $1 \mathrm{mJy}$ sensitivity $(1 \sigma)$ with a daily cadence. As in the case of
ASKAP, we also consider time-averaged versions of the data reaching $1 \sigma \mathrm{rms}$ sensitivities of 0.3 and $0.1 \mathrm{mJy}$ for cadences of 10 days ('CMB-Stack-1') and 100 days ('CMB-Stack-2'), respectively.

One significant drawback of the wide-field CMB instruments is that they have poor angular resolution (14 arcmin for the instrument considered here). This limits both the ability to identify non-varying point sources due to confusion (e.g., Blain et al. 2002) and the quality of event localization. However, the excellent stability of CMB telescopes allows the detection of variable sources far below the confusion limit, and since synchrotron transients evolve from high to low frequencies with time, follow-up of $\mathrm{mm}$ transients with other facilities at $\mathrm{cm}$ wavelengths could lead to better localizations.

\subsection{Square Kilometer Array}

The planned Square Kilometer Array (SKA) will be the most powerful radio telescope ever built (Carilli \& Rawlings 2004). The observatory will be located in both Australia and South Africa and will initially be composed of arrays of three distinct telescope designs denoted SKA1low, SKA1-mid, and SKA1-survey. We consider two hypothetical surveys for transients that could be conducted with the SKA1-survey array, which is planned to have a field of view of $18 \mathrm{deg}^{2}$ and to operate in a frequency range of $0.65-1.67 \mathrm{GHz}$. The first ('SKA') assumes that 100 hours will be spent every quarter in a survey of $10^{4}$ $\operatorname{deg}^{2}$, resulting in a $1 \sigma \mathrm{rms}$ sensitivity of $9 \mu \mathrm{Jy}$. The second ('SKA-Expanded') assumes that the SKA1-survey array will be fully dedicated to a continuous transient survey of $3 \times 10^{4} \mathrm{deg}^{2}$ with a monthly cadence, resulting in a sensitivity of $6 \mu \mathrm{Jy}(1 \sigma)$. This hypothetical survey defines a practical upper limit to what untargeted $\mathrm{GHz}$ transient surveys may be expected to yield in the coming decades. Finally, we consider a low-frequency survey at $150 \mathrm{MHz}$ ('SKA-Low') assuming observations of $10^{4}$ $\operatorname{deg}^{2}$ with 100 hours per epoch on a quarterly cadence, resulting in a sensitivity of $4 \mu \mathrm{Jy}(1 \sigma)$.

\section{SIMULATED TRANSIENT SURVEYS}


In this section we combine the radio light curves and volumetric rates of the transient sources described in $\S 2$ with the survey parameters defined in $\S 3$ to assess the detection rates of various transients, as well as some of their basic properties (e.g., redshift distribution), using Monte Carlo simulations.

\subsection{Detection Criteria}

Our basic criteria for the detection and identification of a transient source are straightforward: (1) a $\gtrsim 10 \sigma$ detection during at least one observing epoch; and (2) at least a factor of 2 change in brightness during the course of the survey (detection or $5 \sigma$ upper limit). The first criterion is motivated by the significant potential for spurious signals given the experiences of untargeted radio transient surveys to date (Gal-Yam et al. 2006; Ofek et al. 2010; Croft et al. 2011; Frail et al. 2012), as well as by the large number of beams and epochs comprising each survey (e.g., $\sim 10^{9}-10^{11}$ beams per epoch for the ASKAP, VLA, and SKA surveys). The second criterion effectively defines the distinction between sources that can be identified as genuine transients during the survey, and those that evolve too slowly to be distinguished from steady or mildly variable sources. Indeed, some variable radio sources can vary by about a factor of two on slow timescales potentially leading to contamination if a robust variability criterion, such as the one we use here, is not utilized (Becker et al. 2010; Ofek et al. 2011).

We note that some previous works on the detectability of radio transients have utilized $5 \sigma$ as a fiducial detection threshold (e.g., Carilli et al. 2003; Bower et al. 2007). Such a low threshold will naturally increase the predicted number of transient detections. However, we do not consider this a realistic threshold since even in the idealized case of purely Gaussian noise in wide-field radio maps, this will lead to an unwieldy $\sim 10^{3}-10^{5}$ false detections per epoch with ASKAP, VLA, and SKA; non-Gaussian effects (e.g., Frail et al. 2012) may increase the number of false detections significantly. Moreover, a $5 \sigma$ threshold reduces the dynamic range for detecting the actual appearance and/or disappearance of a transient, and hence the ability to separate transients from variable and steady sources. Finally, the reduced dynamic range and low signal-to-noise ratio will also inhibit the ability to infer the physical properties and classification of the transients (explosion time, energy, ambient density, velocity, collimation). Thus, we caution that even if the goal of a survey is to simply count the number of radio transients (e.g., Figure 3) the detection threshold should be $\sim 10 \sigma$.

In principle a transient that does not reach a $10 \sigma$ threshold in any single epoch could be detected at somewhat lower significance in multiple epochs and hence be recovered. We essentially account for this effect through our investigation of the "stacked" surveys (VAST-WideStack, VAST-Deep-SF-STACK, CMB-Stack-1, CMBStack-2) in which we carry out the transient detection in temporally-binned versions of the nominal daily cadence data. On the other hand, for the various surveys with a yearly cadence (e.g., VAST-Deep, VLASS), which is comparable to or longer than the typical transient durations (§2.1), such time-averaging is not feasible and $10 \sigma$ remains a robust single-epoch threshold to suppress a large number of false detections.
We emphasize that we do not explicitly model the complex processes of imaging interferometric data, cataloging sources, and measuring fluxes, which are subject to a variety of systematics that have proven challenging to cope with (e.g., radio-frequency interference, spurious sidelobe sources, correlator bugs). Instead we assume that these effects can be approximated as a degradation of a survey's limiting flux density and/or effective area. Accounting for these systematic effects is part of our motivation for choosing $10 \sigma$ as a robust detection threshold. We also assume that each survey achieves its characteristic sensitivity uniformly on the sky, while in practice the sensitivity is spatially variable, or, equivalently, the effective area is a function of sensitivity (e.g., Croft et al. 2013; Williams et al. 2013).

The transient detection criteria in radio surveys should also include the effects of contamination by radio emission from star-forming host galaxies. This has the potential to be more of a problem for radio surveys as compared to optical ones because of the challenges in subtracting sources with complex substructure in interferometric data with sparse $u v$ coverage. Transient searches will likely be performed using flux measurements that include a contribution from the host galaxy, which will reduce the effective change in brightness of a transient.

Host galaxy contamination is of greatest concern for sources that occur exclusively in star-forming galaxies (LGRBs, LLGRBs, Type Ib/c SNe), but it will also affect some fraction of transients that occur in all galaxy types (NS-NS mergers, SGRBs, TDEs). Galaxy contamination is also a greater problem for lower luminosity transients (e.g., LLGRBs, Type Ib/c SNe) than for those that easily outshine their host galaxies (e.g., on-axis LGRBs). In Figure 2, we mark the host galaxy flux density corresponding to a star formation rate of $\mathrm{SFR}=1 \mathrm{M}_{\odot} \mathrm{yr}^{-1}$ :

$$
F_{\nu, \mathrm{gal}} \approx 0.3 \mathrm{mJy} \nu_{\mathrm{GHz}}^{-0.7} D_{L, 27}^{-2}\left(\frac{\mathrm{SFR}}{M_{\odot} \mathrm{yr}^{-1}}\right),
$$

where we use the mean normalization measured for local galaxies (Carilli \& Yun 1999) and adopt a spectral index of -0.7 relevant for galactic synchrotron emission which dominates in this frequency range ${ }^{8}$ (Condon 1992). From Figure 2 it is clear that galaxy contamination will affect a large fraction of transient classes at $150 \mathrm{MHz}$, but that only LLGRBs, Type Ib/c SNe, NSMs, and off-axis SGRBs will be affected at 1-150 GHz.

The host galaxy contamination will be mitigated for sources that are sufficiently nearby such that their hosts will be spatially resolved by the survey interferometer. However, with the exception of the VLASS and the SKA $1.3 \mathrm{GHz}$ surveys, the other surveys have large beam sizes of $\sim 10^{\prime \prime}$ (VAST and SKA-Low), $\sim 1^{\prime}$ (LOFAR), and $\sim 14^{\prime}(\mathrm{CMB})$. For a typical galaxy size of a few kpc this means that the hosts will only be significantly resolved for VAST and SKA-Low within only a small distance of $\lesssim 50 \mathrm{Mpc}$, while for LOFAR and the CMB surveys the hosts will generally be unresolved. Taking into account the transient luminosities, the survey angular resolutions, the expected host galaxy brightness at the var-

8 The $150 \mathrm{MHz}$ flux derived this way may be over-estimated by a factor of $\sim 2$ due to the presence of free-free absorption (e.g., Williams \& Bower 2010). 
ious frequencies, and the occurrence rate of transients in star-forming galaxies we indicate in Tables 3-8 whether host galaxy contamination is expected to be a problem for transient detection.

\subsection{Monte Carlo Method}

Our method for calculating the detection rates and measured properties of the various transients with a given survey is as follows. We create a large number $\left(\gtrsim 10^{4}\right)$ of mock transient light curves using the models in $\S 2$ (Figure 2 ), with random time phasing relative to the start of the survey and with random distances, weighted by the comoving cosmological volume and, when appropriate, star formation rate or SMBH density as described in $\S 2$. The transient light curves are calculated at each redshift, taking into account $\mathrm{K}$-corrections and time dilation effects when necessary. The detection significance at each epoch is determined for each event and survey combination, and the detection criteria are applied to determine the sample of detected events. Absolute detection rates are finally determined by weighting the mock sample by the volumetric event rate and the survey duration. A new realization of events is considered for each survey separately. For each survey we also quantify the fraction of events $\left(f_{V}\right)$ that pass the flux threshold criterion but are miseed by the factor of two variability cut. This fraction provides a measure of how many transients could in principle be recovered with a much longer survey duration.

We note that the issue of detection alone is separate from the question of what qualities make a given event scientifically useful. We defer a detailed discussion of this issue to Paper II. However, some insight into this question can be gained based on a few properties that are readily available from the mock transient sample. In particular, for the transient sources that pass the detection criteria, we determine the number of epochs with $\gtrsim 3 \sigma$ detections to assess the number of light curve data points. We also determine whether the source is detected to rise and/or decline by at least a factor of two relative to the measured light curve peak. If a source is only detected to rise or to decline during the survey, then the only possible measurement is a lower limit on the peak flux and on the rise or decline time, respectively. If the transient is detected at greater than half of its maximum flux at either the first or last epoch of the survey, then the rise or decline time cannot be determined, respectively. These operational definitions are important because unlike in the case of radio follow-up of transients discovered at other wavelengths (e.g., GRBs, SNe, TDEs), the initial time of the event is not known a priori. In Figure 5 we present the cumulative redshift distributions of the transients discovered by the SKA surveys.

\section{SIMULATION RESULTS}

The results of our Monte Carlo simulations are summarized in Tables 3-8 and Figures 3-5. The Tables provide the number of detected transients for each class and survey $(N)$, the mean number of epochs at which a detected transient has a flux density of $\gtrsim 3 \sigma(\bar{n})$, the mean redshift of the detected sources $(\bar{z})$, the fractions $f_{\text {rise }}$ and $f_{\text {fall }}$ of detected transients with "measured" rise and decline times (as defined in $\$ 4.2$ ), respectively, and the fraction of detected transients with their peak occurring during the survey, $f_{\text {peak }}$. For each transient class and survey we also estimate whether galaxy contamination will be an issue ('Yes', 'No', or 'Maybe'). These designations are based on the transient peak brightness relative to the host galaxy radio emission (for a fiducial $\mathrm{SFR}=1$ $\left.\mathrm{M}_{\odot} \mathrm{yr}^{-1}\right)$, as well as on whether all events in a given class are expected to occur in star-forming galaxies. Figure 4 summarizes the total number of detections for each transient class by each survey.

Before addressing the individual results in detail, we consider in Figure 3 the number of sources detected across the entire sky at a fixed point in time as a function of the survey depth $(10 \sigma)$ for each radio frequency and transient class. The number of sources above a given flux density is estimated according to:

$$
N_{\text {all-sky }}\left(>F_{\nu}\right)=\int_{0}^{z\left(F_{\nu, \mathrm{p}}\right)} \frac{\mathcal{R}\left(z^{\prime}\right) t_{\mathrm{dur}}\left(z^{\prime}\right)}{1+z^{\prime}} d V^{\prime}
$$

where $\mathcal{R}\left(z^{\prime}\right)$ is the co-moving volumetric density, $t_{\mathrm{dur}}\left(z^{\prime}\right)$ is the transient duration at redshift $z$ (estimated as the timescale over which the flux is greater than one half of its peak value), the $(1+z)^{-1}$ factor accounts for time dilation of the event rate, and the integral is performed over the co-moving volume $V$ out to the redshift $z\left(F_{\nu}\right)$ corresponding to the peak flux density $F_{\nu, \mathrm{p}}$. We stress that since this is a snapshot rate no variability cuts are applied, and therefore there is no guarantee that all of these sources can actually be identified as transients in a real survey ${ }^{9}$.

As can be seen in Figure 3 all transient classes follow the relation $N_{\text {all-sky }} \propto F_{\nu}^{-3 / 2}$ at high flux densities, consistent with the expectation for Euclidean geometry and a homogeneous source population. However, significant deviations are seen at low flux densities due to a combination of K-corrections, non-Euclidean luminosity distance, and cosmological evolution of the source population. These effects have not been systematically considered in previous studies. For luminous sources, $N_{\text {all-sky }}\left(>F_{\nu}\right)$ is seen to flatten below a critical flux density, corresponding to the depth at which essentially all events are visible to the edge of the Universe; for such sources deeper searches are ineffective at increasing the detection rate. Whether the slope of the distribution flattens or steepens approaching this plateau depends on the importance and sign of the K-correction. An upward steepening of $N_{\text {all-sky }}\left(>F_{\nu}\right)$ occurs for sources with a strong negative K-correction, which is due to the steep positive spectrum of synchrotron self-absorption $\left(F_{\nu} \propto \nu^{2}\right.$ or $\left.\propto \nu^{5 / 2}\right)$; e.g., on-axis LGRBs at $\lesssim 3 \mathrm{GHz}$. On the other hand, a flattening prior to the plateau occurs when the spectrum is flat or inverted; e.g., on-axis LGRBs at $150 \mathrm{GHz}$.

Shown for comparison in Figure 3 are available constraints from previous radio surveys, as well as from the surveys we simulate here. The detection limits are calculated at the $95 \%$ confidence level for a fiducial transient duration of $\sim 100$ days (as motivated in $\S 2.1$ ). The

9 For instance, at $1.3 \mathrm{GHz}, N_{\text {all-sky }}\left(>F_{\nu}\right)$ is at least an order of magnitude larger for the NSM-magnetar case than all other events, but we do not predict a commensurate number of discoveries in actual surveys, because $\sim 75 \%$ of these events do not vary substantially over the survey duration (Table 7 ). 

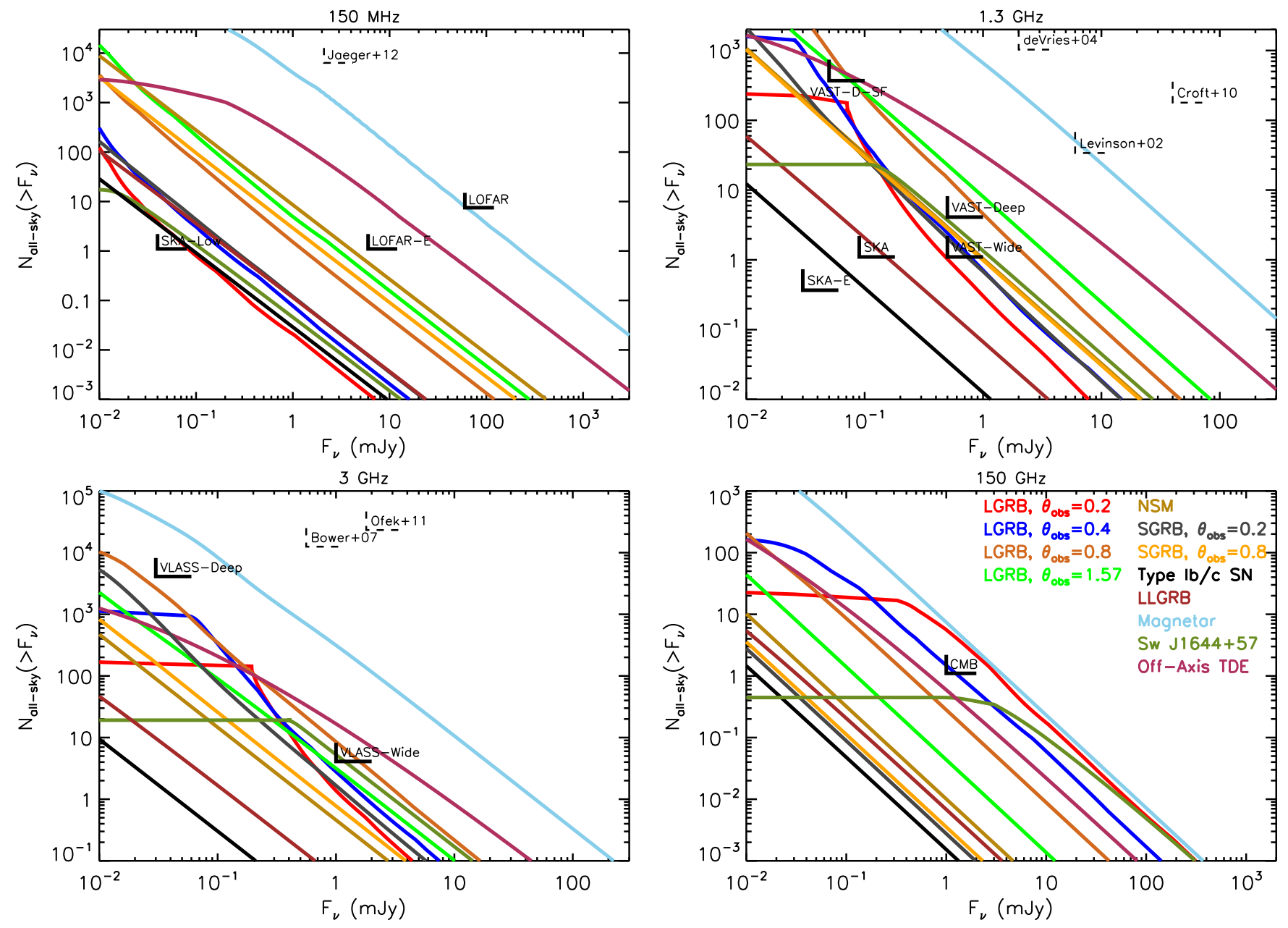

FIG. 3.- Number of sources across the entire sky (eq. [10]) at any time above flux $F_{\nu}$ at observer frequency $\nu=150 \mathrm{MHz}$ (upper left), $1.3 \mathrm{GHz}$ (upper right), $3 \mathrm{GHz}$ (lower right), $150 \mathrm{GHz}$ (lower right). Sources shown include long GRBs $\left(\theta_{\mathrm{obs}}=0.2\right.$, red; $\theta_{\mathrm{obs}}=0.4$, blue; $\theta_{\text {obs }}=0.8$, brown; $\theta_{\mathrm{obs}}=1.57$, green), neutron star merger leaving black hole (olive), NSM-magnetar (light blue), on-axis SGRB (grey), off-axis SGRB (orange), on-axis TDE (Sw J1644+57; dark green), off-axis TDE (purple). Shown for comparison are representative limits placed by the surveys discussed in this paper (Table 2) as well as those placed by past surveys (Appendix A; references: Jaeger et al. 2012b,de Vries et al. 2004, Croft et al. 2010b, Levinson et al. 2002, Gal-Yam et al. 2006, Ofek et al. 2010, Ofek et al. 2011, Bower et al. 2007; see also Frail et al. 2012).

methodology for calculating the upper limits, and a summary of the results from past surveys are given in Appendix A. With the possible exception of NSM-magnetar events $(\S 2.2 .5)$, which at our fiducial rates approach the constraint at $1.4 \mathrm{GHz}$ set by FIRST/NVSS (Levinson et al. 2002), none of the previous surveys reach the required depth/sky area to detect any of the known classes of extragalactic transients, consistent with the lack of confirmed transients to date (Frail et al. 2012).

\subsection{LGRBs}

The VAST (Deep, Wide, and Wide-Stack), VLASS, CMB, and SKA surveys all detect at least one LGRB.

The wide/shallow surveys VAST-Deep, VAST-WideStack, and VLASS-Wide detect $\sim 1-2$ on-axis LGRB afterglows, but this number increases to $\sim 15-30$ for off-axis events. The off-axis events are generally detected over a few epochs, with a low mean redshifts, $\bar{z} \sim 0.1$. In addition, $\sim 50-80 \%$ will have measured rise and decline times. These $\mathrm{GHz}$ surveys should thus produce a reasonably complete sample of LGRB afterglows which are located sufficiently nearby to possess detectable $\mathrm{SNe}$ (detected archivally since the SN optical emission will probably have faded by the time of the first radio detection). However, we note that the SNe may be identified first in the optical, in which case targeted radio follow-up of such events presents a more profitable approach than an untargeted wide-field radio search.

The wide/shallow surveys detect a greater number of LGRBs than the deeper, pencil-beam surveys VLASSDeep and VAST-Deep-SF-Stack, which lead to $N \sim 1-4$ off-axis events. However, the latter do better than predicted from a homogeneous source population because the LGRB volumetric rate increases by an order of magnitude at the larger redshifts $(z \gtrsim 1)$ to which these surveys are sensitive.

The stacked CMB surveys detect $N \approx 40$ events with an average redshift of $\bar{z} \approx 0.7$. The long effective cadence ( 100 days) of the CMB-Stack-2 survey compared to the typical LGRB afterglow duration at $150 \mathrm{GHz}$ renders most events detectable at only 1 epoch, but each detection by the CMB-Stack-1 survey ( $\sim 10$ day cadence) will occur over $\sim 3$ epochs. In the latter case, the explosion time of the GRB will typically be constrained to 


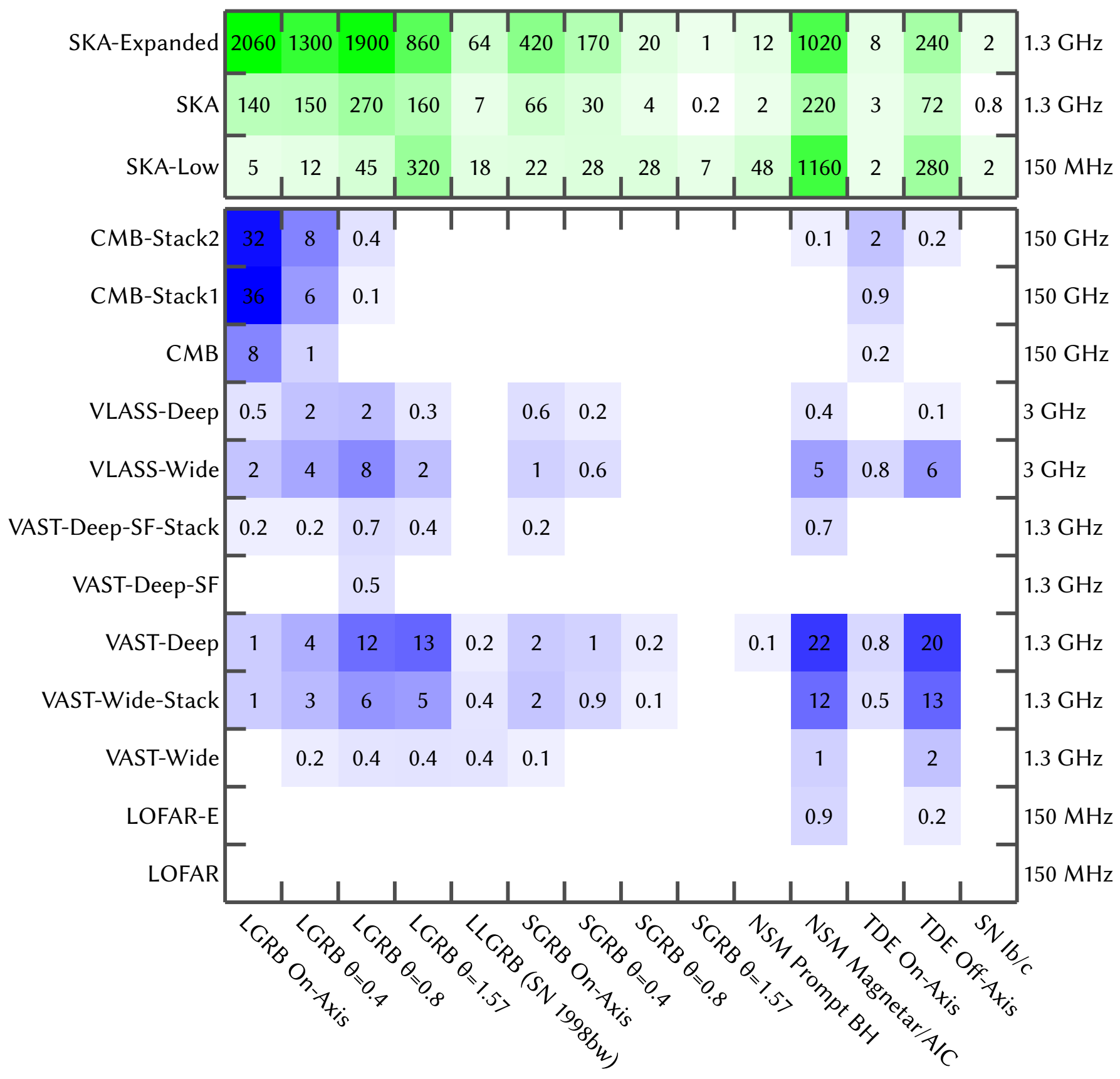

Fig. 4.- Predicted number of event detections ( $N$ in Tables 3-8) by survey and event class. Predicted values less than 0.1 are not shown. Because of the varying quality of constraints on the underlying rate and light curve models, uncertainties in $N$ vary from one event class to another $(\S 2.2)$.

$\lesssim 10$ days, sufficiently early to trigger optical follow-up and lower-frequency radio observations to confirm the afterglow origin and to better localize the event. LGRBs are sufficiently rare and bright at $\bar{z} \approx 0.7$, that the detection or non-detection of $\gamma$-rays from the sky location and explosion time window could constrain the existence of on-axis afterglows that are unaccompanied by a GRB (so-called "dirty fireballs") given the bias towards detecting on-axis events at high observing frequencies.

The number of on-axis LGRBs detected by millimeter surveys may be underestimated since we have only modeled emission from the forward shock, neglecting contributions from the reverse shock which are important at early times. At $150 \mathrm{GHz}$ the reverse shock emission could be a factor of $\gtrsim 10$ times brighter than the forward shock, but lasting for only $\sim 1$ day (e.g. Laskar et al. 2013). Approximately 8 on-axis TDEs were detected by the 1-day cadence $\mathrm{CMB}$ survey to redshift $\bar{z} \sim 0.34$ considering only the forward shock emission. From Figure 2, the number of events in the survey with detectable reverse shock emission could thus be a factor $\sim 20$ times larger, and extending to much higher redshifts $\bar{z} \gtrsim 2$. Alternatively, LGRBs that are detected by the stacked CMB surveys via their forward shock emission could be searched at early survey epochs for reverse shock emission, providing tighter constraints on the time of the GRB and a 

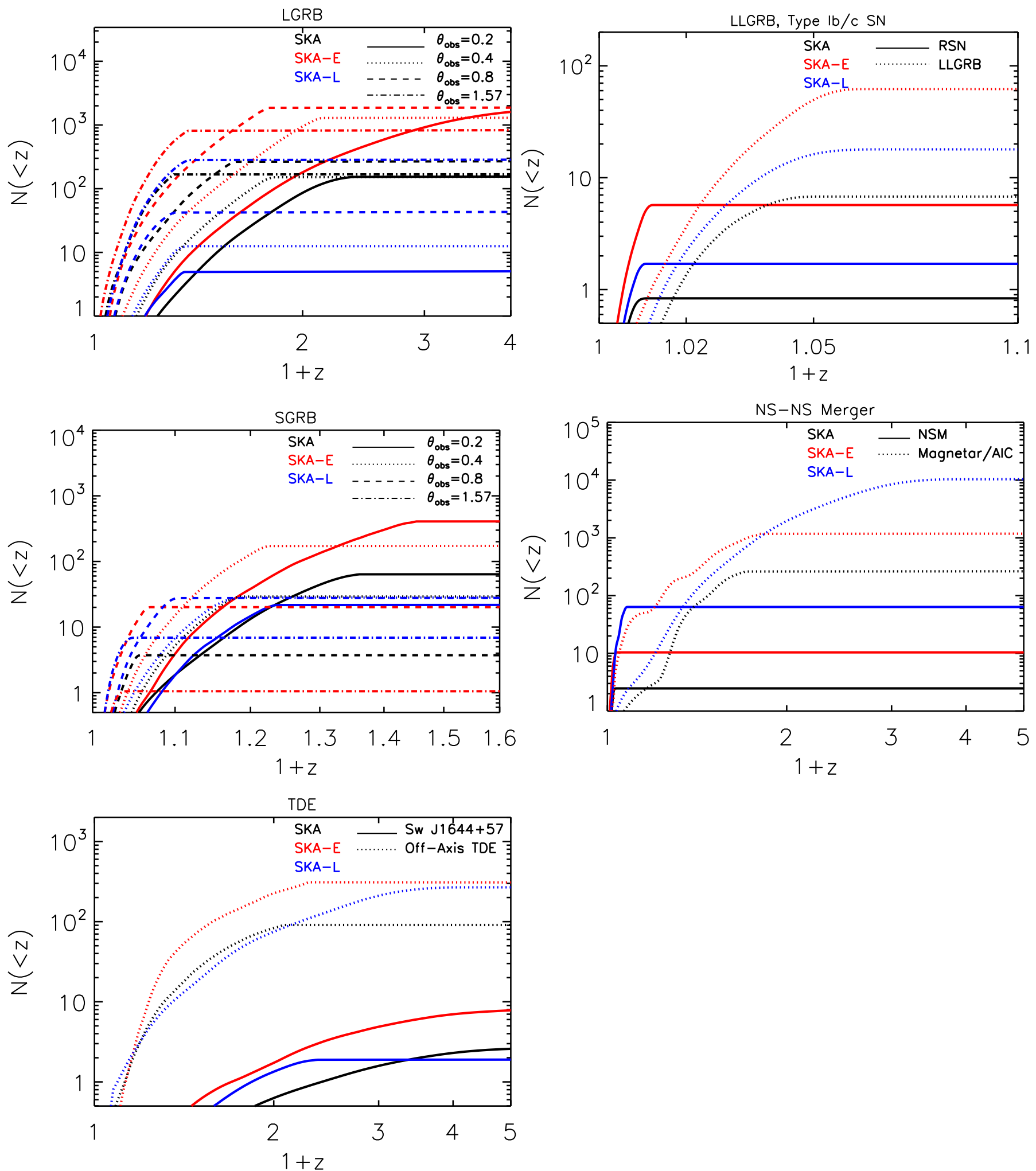

Fig. 5.- Cumulative number of detected sources with redshift less than $z$ for each of the three SKA surveys considered in this paper (SKA: black, SKA-Expanded: red, SKA-Low: blue). Different source classes are shown in different panels and with different line styles as shown in the legends. Note that the top right panel shows results for both LLGRBs and SNe Ibc. 
probe of the magnetization of the ejecta (e.g. Giannios et al. 2008).

The large SKA and SKA-Expanded surveys at $1.3 \mathrm{GHz}$ detect $\sim 700$ and $\sim 6000$ LGRBs, with about $1 / 3$ of the events viewed on-axis. On-axis events represent a larger fraction as compared to VAST/VLASS since they are detected to a higher average redshift, $\bar{z} \sim 1-2$, where the LGRB volumetric rate is an order of magnitude larger than locally, while the off-axis events are still mainly nearby $(\bar{z} \sim 0.5$; Figure 5$)$. SKA-Expanded can detect on-axis LGRBs out to $z>3$. The light curves of most detected sources are reasonably well-sampled, with $\bar{n} \sim 3-27$ epochs, depending on the survey and viewing angle. SKA-Low detects $\sim 400$ LGRBs, mostly far off-axis $\left(\theta_{\text {obs }} \sim \pi / 2\right)$ and originating from $\bar{z} \lesssim 0.3$.

We note that our predicted off-axis LGRB detection rates are approximately a factor of $\sim 3$ times smaller than those predicted by Ghirlanda et al. (2014), who find that ASKAP VAST should have a detection rate of $\sim 3 \times 10^{-3} \mathrm{deg}^{-2} \mathrm{yr}^{-1}$, corresponding to $\sim 90$ events for $10^{4} \operatorname{deg}^{2}$ and a 3 year survey duration. This discrepancy results largely from our difference in detection threshold: Ghirlanda et al. (2014) adopt a $5 \sigma$ detection threshold, which leads to a factor of $\sim 3$ times higher predicted rate.

\section{2. $L L G R B s$}

The SKA surveys are the only ones expected to detect LLGRBs (Table 4 and Figure 4). This is due to the low luminosity of these events $\left(\sim 10^{2}-10^{3}\right.$ times dimmer than off-axis LGRB afterglows), which cannot be overcome by their order of magnitude higher volumetric rate. SKA-Low and SKA-Expanded detect $\sim 20$ and $\sim 60$ LLGRBs at $D_{L} \lesssim 150 \mathrm{Mpc}$ (Figure 5). However, host galaxy contamination could be crippling in the case of SKA-Low due to its relatively poor angular resolution of $11^{\prime \prime}$, coupled with the high galaxy flux density at $150 \mathrm{MHz}$. We further note that at the mean distance of $150 \mathrm{Mpc}$, the broad-lined Type Ib/c SNe, which thus far appear to accompany all LLGRBs, will be discovered independently much earlier in the optical. On the other hand, radio-discovered LLGRBs without bright accompanying $\mathrm{SNe}$ will be of great interest.

\section{3. $S G R B s$}

At most 2-3 SGRBs are detected by the wide/shallow surveys including VAST-Wide-Stack, VAST-Deep, and VLASS-Wide, with a high probability of being close to on-axis $\left(\theta_{\text {obs }} \lesssim 0.4\right)$. No events are detected at larger offaxis angles. Much larger numbers, $\sim 100,90,600$ are detected by the SKA, SKA-Low, and SKA-Expanded, respectively. These events are detected in a modest number of epochs ( $n \sim 3-10)$, providing reasonably wellconstrained rise and decline times.

The mean redshift of the SGRBs detected by the SKA surveys, $\bar{z} \sim 0.2$, is sufficiently high that most events will not have associated gravitational wave detections with Advanced LIGO/Virgo (see also Metzger \& Berger 2012, who reach a similar conclusion based directly on the local SGRB rate). For the few events within the gravitational wave detection range, the (albeit poor) sky localizations from Advanced LIGO/Virgo will be sufficient to uniquely associate the gravitational wave source with the SGRB radio afterglow, even given a $\sim$ year uncertainty in coincidence time.

\subsection{NS-NS Mergers with Prompt BH Formation}

VLASS-Wide-Stack, VAST-Deep, and VLASS-Wide detect $\lesssim 0.1$ NS-NS mergers, although this number is uncertain by at least an order of magnitude given the uncertain local merger rate. These numbers are suppressed by an order of magnitude because a substantial fraction $\left(f_{\mathrm{V}} \sim 0.8-0.9\right)$ of nominal detections are lost to the variability cut, due to the slow evolution of NS-NS merger light curves relative to the survey durations (Figure 2). SKA and SKA-Expanded detects $\sim 2$ and $\sim 10$ events, respectively, with $\bar{z} \sim 0.02$, while SKA-Low detects $\sim 50$ with $\bar{z} \lesssim 0.05$. All detected events are sufficiently close for Advanced LIGO/Virgo to detect their gravitational wave emission, which will happen well in advance of the radio light curve maxima.

\subsection{NS-NS Mergers with Stable Magnetar Remnants 83 White Dwarf AIC}

NSM-magnetar events are among of the most promising transient classes due to their high luminosities. The wide/shallow surveys VAST-Wide-Stack, VAST-Deep, and VLASS-Wide detect $\sim 10,20,5$ events, respectively to an average redshift of $\bar{z} \sim 0.2-0.4$. Due to the long timescales of these events, almost all detections occur at every epoch, and the peak is measured in only $\sim 30-60 \%$ of the cases. SKA, SKA-Expanded, and SKA-Low detect $\sim 200,1000,1200$ events, respectively, to much higher redshifts of $\bar{z} \sim 0.8-1.4$. Unlike the shorter-wavelength surveys, SKA-Low has the capability to detect NSMmagnetar events to $z \gtrsim 2$ (Figure 5 ). We stress that while the optimistic detection rates make these sources of great interest for future radio surveys, the fraction of mergers that lead to stable magnetars (as well as the overall NS-NS merger rate) is highly uncertain. Upcoming surveys will constrain the true occurrence rate.

NSM-magnetar represent the kind of source for which untargeted radio transient surveys have more discovery potential (Metzger \& Bower 2014) because they are expected to be radio-luminous without having counterparts that are more readily discovered at other wavelengths. These events may also be accompanied by luminous Xray and optical emission following the merger or AIC (e.g., Metzger et al. 2008; Yu et al. 2013; Metzger \& Piro 2014), but the short duration and low luminosities of these putative emission processes will make their discovery challenging as compared to at radio frequencies.

\subsection{On-Axis Jetted TDEs (Sw J1644+57)}

CMB-Stack-2 detects $\sim 2$ on-axis TDE, with $\bar{z} \sim 1$, while the wide/shallow GHz surveys VAST-Wide-Stack, VAST-Deep and VLASS-Wide detect $\lesssim 1$ on-axis TDEs (Table 8 ). While the peak flux density of these events is higher at $3 \mathrm{GHz}$ than at $1.3 \mathrm{GHz}$, VLASS loses a greater number of events to the variability cut $\left(f_{\mathrm{V}} \sim 0.5\right)$ as compared to VAST $\left(f_{\mathrm{V}} \sim 0.3-0.4\right)$. The pencil beam survey VLASS-Deep detects fewer events than would be predicted even assuming a Euclidean geometry and homogeneous source population because on-axis TDEs are so luminous that their detection rate is limited not by the flux density threshold of the survey, but by the decreasing SMBH density at $z \gtrsim 4$ and the greater loss of 
events due to the variability cut introduced by time dilation $\left(f_{\mathrm{V}}=0.75\right)$. The same effect limits the number of detections by the various SKA surveys to $\lesssim$ few, despite their much greater sensitivity. In essence, on-axis jetted TDEs are rare events, even if one can probe the entire observable universe.

The long durations of jetted TDE afterglows result in most events being detected in most survey epochs, e.g., $\bar{n} \approx 7$ for CMB-Stack-2, yet the duration is sufficiently short that most will have measured rise times. Although the initial epoch of jet launching will only be constrained by radio observations to within $\sim 100$ days, the rarity of jetted TDEs implies a good chance to unambiguously associate the radio sources with prompt gamma-ray emission detected by Swift or Fermi, either via a previous trigger or through an archival search (e.g., Cenko et al. 2012). The soft X-ray emission might also still be detectable, as luminous X-rays accompanied Sw J1644+57 for about 500 days after the TDE (Zauderer et al. 2013). We also note that the radio detection rates could be higher than our predictions if not all radio-producing onaxis TDEs are accompanied by luminous X-ray emission, since our rates are derived from X-ray-discovered events. However, the typically high redshift of detected events of $\bar{z} \gtrsim 0.6$ implies that constraining the transient location to the nucleus of the host galaxy with sufficient accuracy to confirm a TDE origin will be challenging, likely requiring follow-up JVLA or VLBA imaging.

\subsection{Off-Axis Jetted TDE}

Off-axis jetted TDEs exhibit higher detection rates, with $\approx 13,20,6$ events detected by VAST-Wide-Stack, VAST-Deep and VLASS-Wide, respectively (Table 8). These events occur at lower average redshift $(\bar{z} \sim 0.2-$ 0.3 ; Figure 5 ) than the on-axis events and are also detected at most epochs of the survey $\left(\bar{n} \approx N_{\text {obs }}\right)$. Detected events are reasonably well-characterized, with rise and decline times determined for $\sim 70 \%$ and $\sim 30 \%$ of events, respectively. The typically large viewing angle with respect to the jet axis and relatively small typical distances imply that VLBI imaging of off-axis TDEs could allow the extended jet structure to be resolved (Giannios \& Metzger 2011; Mimica et al. 2015), potentially providing an independent age for the system.

LOFAR-Extended detects less than one event $(N \approx$ $0.2)$ due in part to the large number of events lost to the variability cut $\left(f_{\mathrm{V}} \approx 0.9\right)$. The millimeter surveys also detect $\lesssim 1$ off-axis TDEs due to the suppressed peak flux at high frequencies as compared to the on-axis case.

We note that our overall TDE detection rates are much lower than those predicted by Frail et al. (2012), primarily because these authors assumed a beaming correction to the Sw J1644+57 rate of $10^{3}$ (instead of $10^{2}$ here). They also assumed that the off-axis TDE light curve at $5 \mathrm{GHz}$ is identical to the on-axis case (Sw J1644+57), whereas at $3 \mathrm{GHz}$ we find that the peak flux density is a factor of 6 times dimmer in the off-axis case (Figure 2). Our assumptions result in a predicted TDE radio detection rate that is two orders of magnitude lower than in Frail et al. (2012).

\subsection{Type $\mathrm{Ib} / \mathrm{c} \mathrm{SNe}$}

No Type Ib/c supernovae are detected in any of the surveys (Table 4), except perhaps the SKA surveys, which may detect a few events. As in the case of LLGRBs, these low detection rates result from the low brightness of these events ( $\gtrsim 10^{3}$ times dimmer than off-axis LGRB afterglow), which cannot be overcome by their higher volumetric rates. Also, as in the case of LLGRBs, host galaxy contamination is a significant problem, especially for SKA-Low.

Although it has been argued that perhaps half of all core-collapse SNe are dust obscured (Horiuchi et al. 2011), the actual fraction will need to be much higher than this $(\sim 90 \%$, i.e., a true core-collapse $\mathrm{SN}$ rate that is 10 times higher than inferred from optical surveys) to allow a meaningful rate measurement from untargeted radio surveys due to the small number of detectable events. Because the low-luminosity events do not evolve rapidly (typical timescales $\gtrsim 100$ days at $1.4 \mathrm{GHz}$ ) and are only detectable in the local universe, targeted searches of local galaxies with a few epochs per year are likely to be much more efficient for investigating the prevalence of obscured event populations (§2.2.8).

\subsection{Summary}

Figure 4 graphically summarizes the expected number of event detections for each source class and survey. We briefly summarize the results of our simulations as follows:

- No transients are expected to be detected with LOFAR (even the hypothetical LOFAR-Expanded survey) due to a mismatch between the survey sensitivity and the low peak luminosities of transients at sub-GHz frequencies (self-absorption). The long durations at these frequencies also lead to many events appearing as steady sources.

- At $\mathrm{GHz}$ frequencies, the wide surveys VAST-WideStack, VAST-Deep, and VLASS-Wide are the only pre-SKA surveys producing more than a handful of detections, including $\sim 15-30$ classical LGRBs (mostly off-axis), $\sim 10-40$ TDEs (mostly off-axis), $\sim 2$ on-axis SGRBs, and $\sim 5-20$ NSM-magnetar events. The deep pencil-beam surveys may detect a few transients. However, even though they are sensitive to events at higher redshifts, where the volumetric rate may be higher (e.g., in the case of LGRBs) and positive K-corrections become important, this is insufficient to overcome their lower étendue compared to the wide surveys.

- The CMB-Stack surveys at $150 \mathrm{GHz}$ detect only on-axis events: $\sim 40 \mathrm{LGRBs}$ and $\sim 2$ jetted TDEs.

- The SKA surveys (in particular our hypothetical best-case SKA-Expanded survey) detect hundreds or thousands of LGRBs (on- and off-axis), off-axis jetted TDEs, and NSM-magnetar events. They also detect smaller numbers $(\sim 10-100)$ of LLGRBs, SGRBs, and NS-NS mergers with prompt black hole formation.

- Essentially none of the surveys (with the exception of a few detections with SKA-Expanded) detect Type Ib/c SNe due to their low luminosity. While core-collapse SNe have been discovered in radio observations, these events are not efficiently discovered in untargeted surveys. 


\section{CONCLUSIONS}

We presented general arguments about the constrained timescale-luminosity relation of extragalactic synchrotron transients, and utilized the light curves for a wide range of known and hypothetical transients and the properties of multiple planned and hypothetical radio surveys spanning $150 \mathrm{MHz}$ to $150 \mathrm{GHz}$ to explore the detection rates and basic properties of radio transients. Our finding can be summarized as follows:

- The basic physics of synchrotron-emitting extragalactic transients place significant constraints on their possible radio light curves. Of particular relevance to radio transient surveys are the facts that luminous events cannot evolve on arbitrarily short timescales, and that the emission evolves from high to low frequencies. Events with radio luminosities sufficient to be detected in appreciable numbers with future radio surveys (e.g., LGRBs, TDEs) have characteristic timescales of $\gtrsim 100$ days at $\mathrm{GHz}$ frequencies and about an order of magnitude longer at $\sim 0.1 \mathrm{GHz}$.

- Our Monte Carlo simulations predict detection rates for planned and hypothetical radio transient surveys, for the first time using an end-to-end model including realistic volumetric rates and light curves, and simulated survey strategies. We account for all relevant cosmological effects, including time dilation, $\mathrm{K}$-corrections, rate evolution, and non-Euclidean luminosity distance, which are important in the context of the most sensitive surveys such as SKA.

- Low-luminosity transients require volumetric rates comparable to the local core-collapse SN rate to be discoverable in untargeted radio transient surveys. They will therefore account for at most a few events in all pre-SKA surveys, and even with SKA will number $\sim 10-100$ in the entire survey. Such transients are therefore best studied through targeted radio follow-up of discoveries from other wavelengths, if they produce emission outside of the radio band. However, even in the case that they produce only radio emission, or are dust-obscured, a more profitable approach may be to target nearby galaxies.

- The small number of expected detections in the pre-SKA surveys (and the hundreds to thousands of detections with SKA) are dominated by relativistic sources (LGRBs, TDEs, NSM-magnetars) both on- and off-axis. These events are detected at cosmological distances where redshift determinations from host galaxies may prove challenging, and the ability to locate them to specific regions of the hosts (e.g., the nucleus in the case of TDEs) may be limited.

- Our hypothetical millimeter surveys will detect mainly on-axis LGRBs and TDEs. The detection rates we calculate may be enhanced if additional events are detected by their reverse shock emission, which is significantly brighter than the forward shock emission but shorter lived. LGRBs discovered by the stacked CMB surveys via their forward shock emission could also be searched at early survey epochs for reverse shock emission.

- Our predicted detection rates are generally lower than previous claims (Figure 4). We predict that the most successful pre-SKA survey will be VASTDeep, with $\sim 70$ extragalactic transient detections across all considered classes. In some cases, the lower numbers that we predict are due to more conservative assumptions about the underlying events (e.g., §5.7). Another factor is our $10 \sigma$ detection criterion, which is generally higher than previous studies, but also more realistic given the scale of the surveys and lessons about false-positive candidates from past searches (Frail et al. 2012). Our simulations also highlight the impact of slow evolutionary timescales at low frequencies which will restrict the ability to separate transient sources from variable and steady sources. We also note that some previous studies have overestimated the effective areas of surveys that observe at cadences significantly higher than the relevant evolution times.

- We find that host galaxy contamination is not expected to be a problem for most detected transients, because the detections are dominated by luminous events that outshine their host galaxies for moderate star formation rates of $\sim 1$ $10 \mathrm{M}_{\odot} \mathrm{yr}^{-1}$. The small numbers of detections of the low-luminosity transient classes will be reduced at $\lesssim \mathrm{GHz}$ frequencies, however, if the host galaxy emission cannot be subtracted cleanly. This is especially relevant for SKA-Low, because at low frequency only a few radio transients (on-axis SGRBs, NSM-magnetars, TDEs) manage to significantly outshine their host galaxies, and the resolution of low frequency transient surveys is relatively poor (11" for SKA-Low).

- For near-term surveys, we find that areal densities of realistic extragalactic radio transients generally follow a Euclidean scaling relation, $N_{\text {all-sky }}\left(>F_{\nu}\right) \propto F_{\nu}^{-3 / 2}$, where $N_{\text {all-sky }}\left(>F_{\nu}\right)$ is number of transients on the sky brighter than some flux density cutoff, $F_{\nu}$ (Figure 3 ). As a rule of thumb, non-Euclidean effects are not significant for transients brighter than $\sim 1 \mathrm{mJy}$ in almost all circumstances, and for some sources they can be ignored down to flux densities of $\sim 10 \mu \mathrm{Jy}$. However, for the more sensitive SKA surveys, which are the only surveys expected to detect a large number of transients, non-Euclidan effects and cosmological source evolution cannot be neglected.

- The small number of predicted events in pre-SKA surveys will inhibit attempts to measure event rates and beaming fractions, since the uncertainties induced by small-number statistics will be significant. It is also unclear if the various transient sources can be distinguished from each other in surveys that span only a small number of epochs.

- The SKA will detect hundreds to thousands of events. These will be dominated by LGRBs but 
will also include SGRBs (primarily on-axis), offaxis TDEs, and NSM-magnetar events.

- NSM-magnetar events have the potential to be a major source of radio transients and may dominate the yield of SKA-Low. However, the underlying rates and light curves are currently highly uncertain; upcoming surveys will constrain them.

We have not considered the question of what astrophysical information can be extracted once a radio transient is discovered. As we have emphasized, the general lag of radio emission behind that at other bands makes the detailed characterization of radio-discovered transients challenging. Paper II will investigate this topic in detail.

This research has made use of the SIMBAD database, operated at CDS, Strasbourg, France, and NASA's Astrophysics Data System. BDM acknowledges helpful conversations with Dimitrios Giannios, Bradley Johnson, Glenn Jones, David Kaplan, Peter Mimica, and Ashley Zauderer. We thank Aleksey Generozov for technical assistance and Rodolfo Barniol-Duran for providing LLGRB light curve models. BDM gratefully acknowledges support from NASA Fermi grant NNX14AQ68G, NSF grant AST-1410950, and the Alfred P. Sloan Foundation. 
TABLE 3

LGRBS

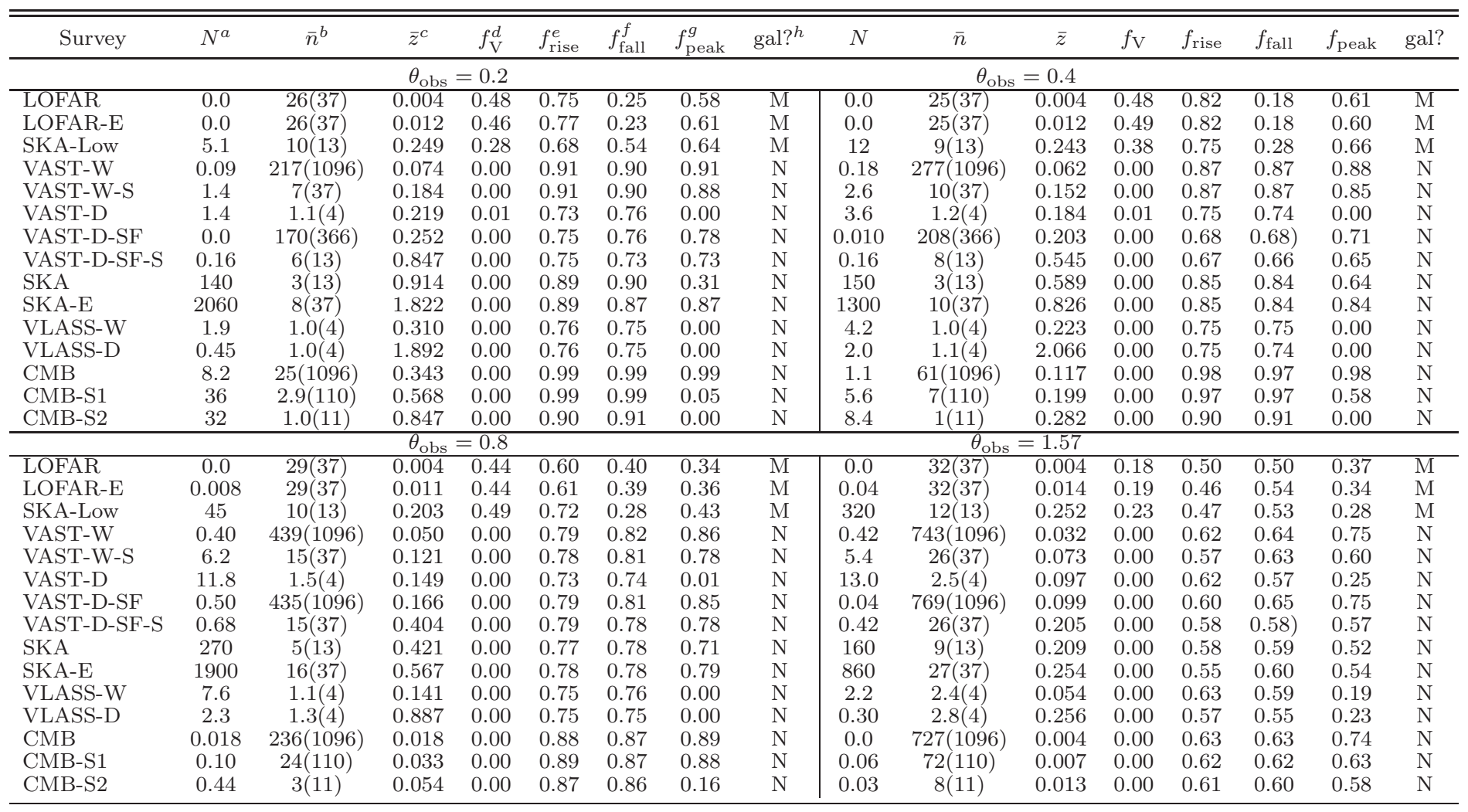

NoтE. - ${ }^{a}$ Total number of detected events. ${ }^{b}$ Average number of detected epochs (total epochs). ${ }^{c}$ Average redshift of detected events. ${ }^{d}$ Fraction of total detected events discarded for lack of variability. ${ }^{e}$ Fraction of events with measured rise times. ${ }^{f}$ Fraction of events with measured fall times. ${ }^{g}$ Fraction of events with a measured peak. ${ }^{h}$ Is galaxy contamination an issue? (Yes, No, Maybe).

TABLE 4

LLGRBS

\begin{tabular}{lcccccccc}
\hline \hline \multicolumn{1}{c}{ Survey } & $N$ & $\bar{n}$ & $\bar{z}$ & $f_{\mathrm{V}}$ & $f_{\text {rise }}$ & $f_{\text {fall }}$ & $f_{\text {peak }}$ & gal? \\
\hline LOFAR & 0.0 & $17(37)$ & 0.001 & 0.00 & 0.82 & 0.86 & 0.81 & $\mathrm{Y}$ \\
LOFAR-E & 0.0 & $17(37)$ & 0.002 & 0.00 & 0.81 & 0.85 & 0.80 & $\mathrm{Y}$ \\
SKA-Low & 18 & $6(13)$ & 0.036 & 0.00 & 0.81 & 0.83 & 0.68 & $\mathrm{Y}$ \\
VAST-W & 0.40 & $140(1096)$ & 0.005 & 0.00 & 0.96 & 0.96 & 0.96 & $\mathrm{Y}$ \\
VAST-W-S & 0.36 & $6(37)$ & 0.011 & 0.00 & 0.95 & 0.95 & 0.30 & $\mathrm{Y}$ \\
VAST-D & 0.18 & $1.2(4)$ & 0.012 & 0.00 & 0.73 & 0.76 & 0.00 & $\mathrm{Y}$ \\
VAST-D-SF & 0.0 & $141(1096)$ & 0.016 & 0.00 & 0.96 & 0.97 & 0.96 & $\mathrm{Y}$ \\
VAST-D-SF-S & 0.03 & $6(37)$ & 0.031 & 0.00 & 0.95 & 0.94 & 0.29 & $\mathrm{Y}$ \\
SKA & 6.6 & $2(13)$ & 0.029 & 0.00 & 0.90 & 0.93 & 0.02 & $\mathrm{M}$ \\
SKA-E & 64 & $6(37)$ & 0.040 & 0.00 & 0.94 & 0.95 & 0.30 & $\mathrm{M}$ \\
VLASS-W & 0.060 & $1.1(4)$ & 0.010 & 0.00 & 0.77 & 0.72 & 0.00 & $\mathrm{M}$ \\
VLASS-D & 0.012 & $1.1(4)$ & 0.053 & 0.00 & 0.74 & 0.77 & 0.00 & $\mathrm{Y}$ \\
CMB & 0.04 & $4(1096)$ & 0.005 & 0.00 & 1.00 & 1.00 & 0.00 & $\mathrm{Y}$ \\
CMB-S1 & 0.034 & $1(110)$ & 0.008 & 0.00 & 1.00 & 1.00 & 0.00 & $\mathrm{Y}$ \\
CMB-S2 & 0.016 & $1(11)$ & 0.012 & 0.00 & 0.89 & 0.89 & 0.00 & $\mathrm{Y}$ \\
\hline
\end{tabular}

Note. - Columns are as in Table 3. 
TABLE 5

SGRBS

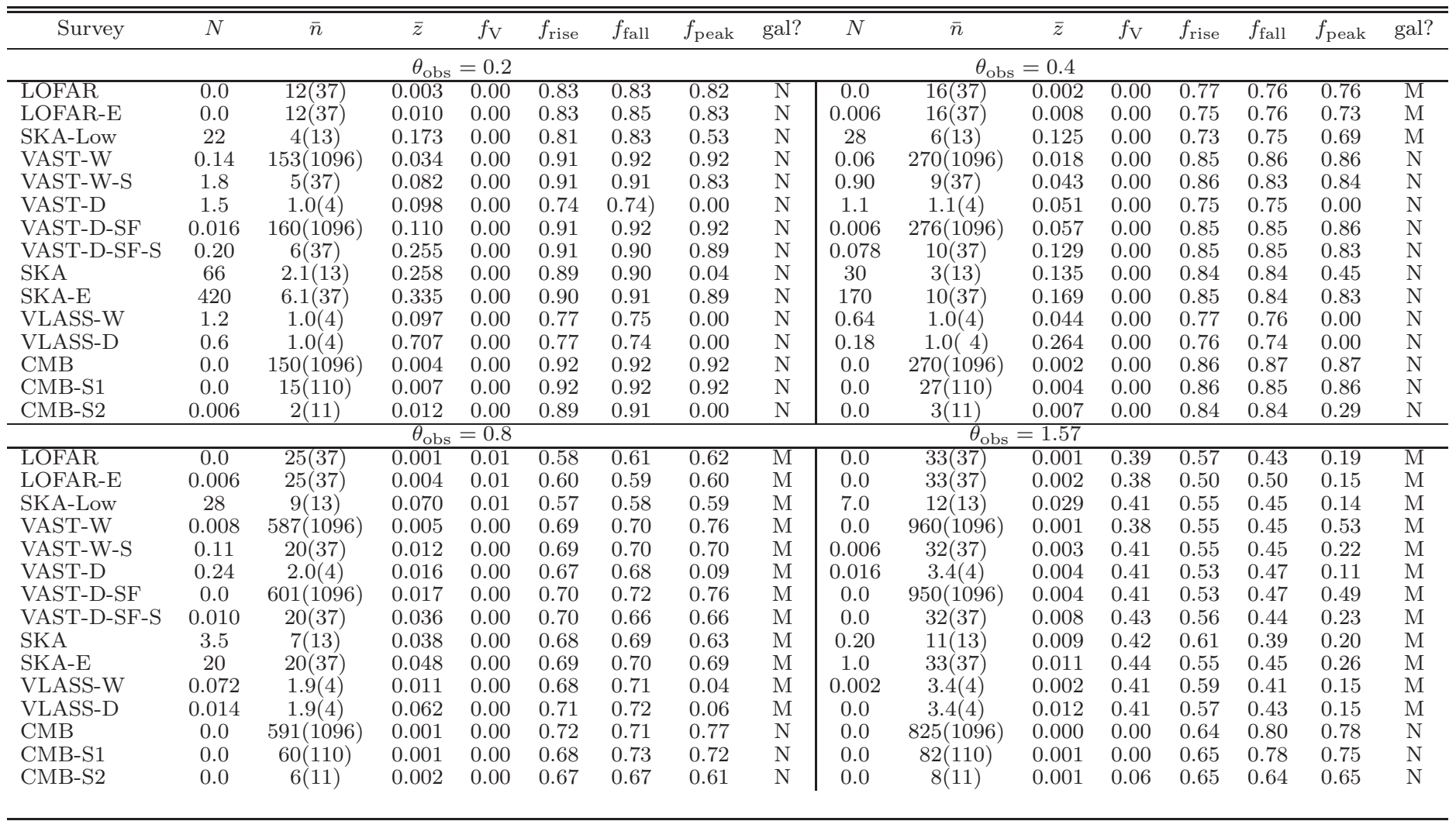

Note. - Columns are as in Table 3.

TABLE 6

NS-NS Mergers with Prompt Black Hole Formation

\begin{tabular}{lcccccccc}
\hline \hline \multicolumn{1}{c}{ Survey } & $N$ & $\bar{n}$ & $\bar{z}$ & $f_{\mathrm{V}}$ & $f_{\text {rise }}$ & $f_{\text {fall }}$ & $f_{\text {peak }}$ & gal? \\
\hline LOFAR & 0.0 & $36(37)$ & 0.001 & 0.82 & 1.00 & 0.00 & 0.23 & $\mathrm{M}$ \\
LOFAR-E & 0.016 & $37(37)$ & 0.003 & 0.81 & 1.00 & 0.00 & 0.21 & $\mathrm{M}$ \\
SKA-Low & 48 & $13(13)$ & 0.046 & 0.84 & 1.00 & 0.00 & 0.04 & $\mathrm{M}$ \\
VAST-W & 0.0 & $1095(1096)$ & 0.002 & 0.87 & 1.00 & 0.00 & 0.33 & $\mathrm{M}$ \\
VAST-W-S & 0.060 & $37(37)$ & 0.005 & 0.86 & 1.00 & 0.00 & 0.15 & $\mathrm{M}$ \\
VAST-D & 0.13 & $3.9(4)$ & 0.008 & 0.86 & 1.00 & 0.00 & 0.00 & $\mathrm{M}$ \\
VAST-D-SF & 0.0 & $1095(1096)$ & 0.007 & 0.80 & 1.00 & 0.00 & 0.30 & $\mathrm{M}$ \\
VAST-D-SF-S & 0.006 & $37(37)$ & 0.015 & 0.84 & 1.00 & 0.00 & 0.13 & $\mathrm{M}$ \\
SKA & 1.9 & $13(13)$ & 0.018 & 0.84 & 1.00 & 0.00 & 0.08 & $\mathrm{M}$ \\
SKA-E & 12 & $37(37)$ & 0.020 & 0.82 & 1.00 & 0.00 & 0.09 & $\mathrm{M}$ \\
VLASS-W & 0.026 & $4.0(4)$ & 0.004 & 0.83 & 1.00 & 0.00 & 0.00 & $\mathrm{~N}$ \\
VLASS-D & 0.0 & $3.9(4)$ & 0.021 & 0.86 & 1.00 & 0.00 & 0.00 & $\mathrm{~N}$ \\
CMB & 0.0 & $1096(1096)$ & 0.000 & 0.80 & 1.00 & 0.00 & 0.48 & $\mathrm{~N}$ \\
CMB-S1 & 0.0 & $110(110)$ & 0.001 & 0.83 & 1.00 & 0.00 & 0.20 & $\mathrm{~N}$ \\
CMB-S2 & 0.0 & $11(11)$ & 0.001 & 0.86 & 1.00 & 0.00 & 0.02 & $\mathrm{~N}$ \\
\hline
\end{tabular}

Note. - Columns are as in Table 3. 
TABLE 7

NS-NS Mergers with a Stable Magnetar Remnant

\begin{tabular}{lcccccccc}
\hline \hline \multicolumn{1}{c}{ Survey } & $N$ & $\bar{n}$ & $\bar{z}$ & $f_{\mathrm{V}}$ & $f_{\text {rise }}$ & $f_{\text {fall }}$ & $f_{\text {peak }}$ & gal? \\
\hline LOFAR & 0.0 & $33(37)$ & 0.038 & 0.76 & 1.00 & 0.00 & 0.00 & $\mathrm{~N}$ \\
LOFAR-E & 0.90 & $33(37)$ & 0.131 & 0.76 & 1.00 & 0.00 & 0.14 & $\mathrm{~N}$ \\
SKA-Low & 1160 & $12(13)$ & 1.425 & 0.86 & 1.00 & 0.00 & 0.15 & $\mathrm{~N}$ \\
VAST-W & 1.2 & $870(1096)$ & 0.140 & 0.58 & 0.95 & 0.05 & 0.73 & $\mathrm{~N}$ \\
VAST-W-S & 12 & $31(37)$ & 0.296 & 0.65 & 1.00 & 0.00 & 0.61 & $\mathrm{~N}$ \\
VAST-D & 22 & $3.2(4)$ & 0.371 & 0.70 & 1.00 & 0.00 & 0.31 & $\mathrm{~N}$ \\
VAST-D-SF & 0.080 & $945(1096)$ & 0.388 & 0.65 & 0.99 & 0.01 & 0.82 & $\mathrm{~N}$ \\
VAST-D-SF-S & 0.72 & $33(37)$ & 0.755 & 0.72 & 1.00 & 0.00 & 0.53 & $\mathrm{~N}$ \\
SKA & 220 & $12(13)$ & 0.805 & 0.76 & 1.00 & 0.00 & 0.50 & $\mathrm{~N}$ \\
SKA-E & 1020 & $34(37)$ & 0.887 & 0.75 & 1.00 & 0.00 & 0.40 & $\mathrm{~N}$ \\
VLASS-W & 5.0 & $3.1(4)$ & 0.227 & 0.63 & 0.98 & 0.02 & 0.39 & $\mathrm{~N}$ \\
VLASS-D & 0.38 & $3.5(4)$ & 0.948 & 0.76 & 1.00 & 0.00 & 0.16 & $\mathrm{~N}$ \\
CMB & 0.006 & $917(1096)$ & 0.024 & 0.47 & 0.77 & 0.23 & 0.72 & $\mathrm{~N}$ \\
CMB-S1 & 0.032 & $92(110)$ & 0.042 & 0.47 & 0.79 & 0.21 & 0.67 & $\mathrm{~N}$ \\
CMB-S2 & 0.13 & $9(11)$ & 0.070 & 0.60 & 1.00 & 0.00 & 0.62 & $\mathrm{~N}$ \\
\hline
\end{tabular}

Note. - Columns are as in Table 3.

TABLE 8

TDES

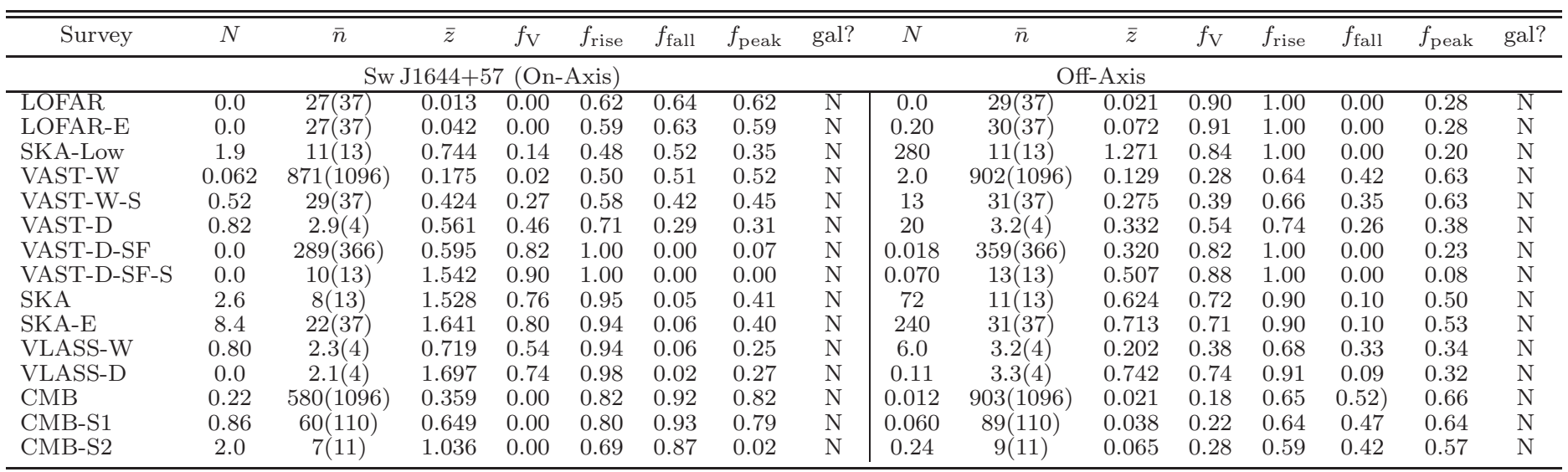

Note. - Columns are as in Table 3.

TABLE 9

TyPe IB/C SNe

\begin{tabular}{lcccccccc}
\hline \hline \multicolumn{1}{c}{ Survey } & $N$ & $\bar{n}$ & $\bar{z}$ & $f_{\mathrm{V}}$ & $f_{\text {rise }}$ & $f_{\text {fall }}$ & $f_{\text {peak }}$ & gal? \\
\hline LOFAR & 0.0 & $30(37)$ & 0.001 & 0.07 & 0.59 & 0.54 & 0.60 & $\mathrm{Y}$ \\
LOFAR-E & 0.0 & $30(37)$ & 0.002 & 0.12 & 0.60 & 0.54 & 0.62 & $\mathrm{Y}$ \\
SKA-Low & 1.6 & $11(13)$ & 0.007 & 0.13 & 0.60 & 0.53 & 0.59 & $\mathrm{Y}$ \\
VAST-W & 0.0 & $386(1096)$ & 0.001 & 0.00 & 0.88 & 0.91 & 0.88 & $\mathrm{Y}$ \\
VAST-W-S & 0.032 & $13(37)$ & 0.002 & 0.00 & 0.88 & 0.90 & 0.86 & $\mathrm{Y}$ \\
VAST-D & 0.036 & $1.8(4)$ & 0.002 & 0.00 & 0.71 & 0.74 & 0.01 & $\mathrm{Y}$ \\
VAST-D-SF & 0.0 & $390(1096)$ & 0.003 & 0.00 & 0.88 & 0.91 & 0.88 & $\mathrm{Y}$ \\
VAST-D-SF-S & 0.0 & $14(37)$ & 0.006 & 0.00 & 0.87 & 0.92 & 0.87 & $\mathrm{Y}$ \\
SKA & 0.85 & $5(13)$ & 0.006 & 0.00 & 0.84 & 0.88 & 0.27 & $\mathrm{M}$ \\
SKA-E & 1.6 & $13(37)$ & 0.008 & 0.00 & 0.87 & 0.90 & 0.85 & $\mathrm{M}$ \\
VLASS-W & 0.010 & $1.4(4)$ & 0.002 & 0.00 & 0.71 & 0.78 & 0.00 & $\mathrm{M}$ \\
VLASS-D & 0.0 & $1.5(4)$ & 0.010 & 0.00 & 0.72 & 0.78 & 0.00 & $\mathrm{Y}$ \\
CMB & 0.008 & $31(1096)$ & 0.001 & 0.00 & 1.00 & 1.00 & 0.22 & $\mathrm{Y}$ \\
CMB-S1 & 0.008 & $12(110)$ & 0.002 & 0.00 & 0.97 & 0.98 & 0.19 & $\mathrm{Y}$ \\
CMB-S2 & 0.006 & $3(11)$ & 0.003 & 0.06 & 0.65 & 0.93 & 0.00 & $\mathrm{Y}$ \\
\hline
\end{tabular}

Note. - Columns are as in Table 3. 


\section{APPENDIX}

\section{LIMITS FROM PREVIOUS SURVEYS}

Previous surveys are placed in Figure 3 according $F_{\nu, \text { lim }}$, to their source detection thresholds ${ }^{10}$, and their constraints of $\mathrm{N}_{\text {all-sky }}$. Below we give sensitivity numbers for these surveys. In the vast majority of cases no sources are detected,

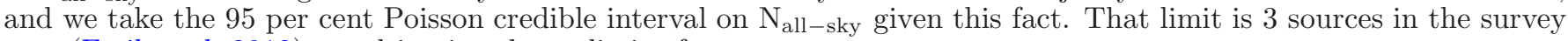
area (Frail et al. 2012), resulting in a lower limit of

$$
N_{\text {all-sky }}<123800\left(\frac{\Omega_{s}}{\operatorname{deg}^{2}}\right)^{-1},
$$

where $\Omega_{s}$ is the effective survey area, which is a function of the transient evolutionary time scale $t_{\text {dur }}$ because repeated visits to a field do not add more information if the time between them is much shorter than $t_{\text {dur }}$ (Macquart 2014). We make the approximation that when new epochs are obtained at a cadence $T_{\text {sep }} \lesssim t_{\text {dur }}$, the effective survey area is

$$
\Omega_{s}=\Omega_{\mathrm{fp}} \frac{T_{\mathrm{bl}}}{t_{\mathrm{dur}}}
$$

where $\Omega_{\mathrm{fp}}$ is footprint area of the survey and $T_{\mathrm{bl}}$ is the total time baseline of the survey; the variability loss factor $V \approx t_{\mathrm{dur}} / T_{\mathrm{bl}}$. A survey with a duration small compared to $t_{\mathrm{dur}}$ has $\Omega_{s} \rightarrow 0$, since separate observations result in zero independent measurements in this case.

Based on the analysis in section 2, here we assume a 100-day timescale in all cases. This means that in many cases our adopted values of $\Omega_{s}$ are significantly smaller than the limiting case of the "two-epoch effective survey area" (Bower et al. 2007):

$$
\Omega_{s, 2 \mathrm{E}}=\Omega_{\mathrm{fp}}\left(N_{\mathrm{ep}}-1\right)
$$

where $N_{\text {ep }}$ is the number of survey epochs, which applies only when $T_{\text {sep }} \gtrsim t_{\text {dur }}$. In the case of ASKAP-Wide, $\Omega_{s}$ as estimated this way is more than two orders of magnitude too large. We note that because both Equations A2 and A3 have $\Omega_{s} \propto \Omega_{\mathrm{fp}} \propto \tau^{-1}$, however, wide and shallow surveys are preferred for Euclidean sources regardless of the relevant time scales.

\section{VLSS (150 MHz)}

Jaeger et al. (2012a) summarize ongoing work to perform a systematic search for transients in the VLA Low Frequency Sky Survey (VLSS; Lane et al. 2012). Jaeger et al. (2012b) report one detection, which occurred in one epoch of a 6 -epoch search for transients in a $6.5 \mathrm{deg}^{2}$ field of view. The detection at $5.5 \sigma(2.1 \mathrm{mJy})$ occurred in the middle of the campaign, which lasted only 3 months. The source appears to rise in flux by a factor of two on a 6 -hr time scale in the detection epoch. Upper limits for the other epochs are not provided by Jaeger et al. (2012b).

As the time scale of this survey is about the same as our 100-day choice, we set the detection density to less than one source per $6.5 \mathrm{deg}^{2}$, corresponding to $N_{\text {all-sky }}<6400$. Note that this is well above the predictions for most of our transient classes. The rapid variability observed in the discovery epoch does not necessarily indicate unrealistically fast timescales, as this may be symptomatic of the low significance of the detection. To summarize, we take $F_{\nu, \mathrm{lim}}=2.1 \mathrm{mJy}$ and $N_{\text {all-sky }}<6400$.

\section{$\operatorname{FIRST}(1.4 \mathrm{GHz})$}

No transients are reported in comparison of the zero-declination strip of the FIRST survey by de Vries et al. (2004), which was visited in two epochs separated by 7 years. The quoted area is $120.2 \operatorname{deg}^{2}$ (their section 2 ) and the source flux density limit is $2 \mathrm{mJy}$ (their section 2.1.1). We take $F_{\nu, \mathrm{lim}}=2 \mathrm{mJy}$ and $N_{\text {all-sky }}<1030$.

\section{ATATS/NVSS $(1.4 \mathrm{GHz})$}

Croft et al. (2010b) compare the Allen Telescope Array Twenty-centimeter Survey (ATATS) with NVSS, reporting no transients with a source flux density limit of $40 \mathrm{mJy}$. As the time baseline between the two surveys is $\gg 100$ days, the effective area is the ATATS footprint of $690 \mathrm{deg}^{2}$. We thus take $F_{\nu, \text { lim }}=40 \mathrm{mJy}$ and $N_{\text {all-sky }}<179$.

\section{$\operatorname{FIRST/NVSS}(1.4 \mathrm{GHz})$}

Levinson et al. (2002) searched for transients in the overlap of the FIRST and NVSS surveys. The effective search area is $2400 \mathrm{deg}^{2}$, and the characteristic limit is $6 \mathrm{mJy}$. Since FIRST and NVSS were conducted quasi-simultaneously, that the time baseline between the two epochs varied between $\sim 0.5$ and $4.5 \mathrm{yr}$. Gal-Yam et al. (2006) followed up the transient candidates from Levinson et al. (2002), concluding that two were real: one radio supernovae and a second object without an apparent host galaxy. Based on our calculations, the RSN detection was a highly improbable result. To summarize, we take $F_{\nu, \lim }=6 \mathrm{mJy}$ and $N_{\text {all-sky }}=34$.

${ }^{10}$ Note that in these works it is not necessarily true that $F_{\nu, \lim }=10 \sigma$, as we have adopted. 


\section{VLA Low-Galactic-Latitude Survey (5 GHz)}

Ofek et al. (2011) report a survey with a footprint of $2.66 \mathrm{deg}^{2}$ comprising 16 epochs. The spacing of the epochs (their Table 3) is such that only 2 of them are independent on 100 -day time scales, so we adopt $\mathrm{A}=5.32$ deg ${ }^{2}$, significantly smaller than the naive effective area of $41 \mathrm{deg}^{2}$. An $8 \sigma$ source was reported that was present in the very first epoch and became undetectable 3 days later. Since this evolution appears unrealistically rapid, this source was probably spurious even though it passed various quality checks, a conclusion shared by other groups (Frail et al. 2012). Ofek et al. (2011) report a representative flux density limit of $1.8 \mathrm{mJy}$ (their section 9.1 ), so we take $F_{\nu, \text { lim }}=1.8 \mathrm{mJy}$ and $N_{\text {all-sky }}=23270$. We note that although we present the limit from this survey with our $3 \mathrm{GHz}$ results, the survey was conducted at $5 \mathrm{GHz}$.

\section{VLA Calibrator Field Survey (5 \& $8 \mathrm{GHz})$}

Bower et al. (2007) report the results of a 944-epoch survey for transient sources with archival data from the VLA spanning 22 years with a typical epoch separation of 7 days. We update the results to account for the improved re-analysis of Frail et al. (2012). At a 100 day cadence, the 22 years of observations represent only 80 independent epochs, so the effective area is reduced by a factor of $\sim 10$ compared to a naive estimate. Observations were performed at 4.9 and $8.4 \mathrm{GHz}$, and again we show this survey with our $3 \mathrm{GHz}$ results.

Frail et al. (2012) conclude that there is only one transient in the data set, with a flux density of $0.6 \mathrm{mJy}$ at $4.9 \mathrm{GHz}$ and a significance of $5.8 \sigma$. It only appears in one epoch. As with the Jaeger et al. (2012b) transient, the detected event is too fast and implies a rate far above our predictions, but we treat it as real. Bower et al. (2007) report effective areas as a function of the flux density limit and the characteristic time scale (their Tables 5-7). We employ the limit closest to the detected transient flux density of $0.56 \mathrm{mJy}$. On a 2-month time scale, their Table 6 gives an effective area of $4.97 \mathrm{deg}^{2}$ with 120 epochs. Scaling by 80/120 (i.e., converting $\Omega_{s}$ to use the definition of Equation A2 rather than Equation A3) gives a corrected effective area of $3.3 \mathrm{deg}^{2}$. We thus take $F_{\nu, \lim }=0.56 \mathrm{mJy}$ and $N_{\text {all-sky }}=12500$.

\section{REFERENCES}

Araujo, D. C., Ade, P. A. R., Bond, J. R., et al. 2014, ArXiv e-prints, arXiv:1407.6249 [astro-ph.IM]

Barniol Duran, R., Nakar, E., Piran, T., \& Sari, R. 2014, ArXiv e-prints, arXiv:1407.4475 [astro-ph.HE]

Becker, R. H., Helfand, D. J., White, R. L., \& Proctor, D. D. 2010, AJ, 140, 157

Becker, R. H., White, R. L., \& Helfand, D. J. 1995, ApJ, 450, 559

Belczynski, K., O'Shaughnessy, R., Kalogera, V., et al. 2008, ApJ, 680, L129

Bell, M. E., Fender, R. P., Swinbank, J., et al. 2011, MNRAS, 415,2

Berger, E. 2002, ApJ, 572, 503

-. 2014, ARA\&A, 52, 43

Berger, E., Kulkarni, S. R., \& Frail, D. A. 2004, ApJ, 612, 966

Berger, E., Kulkarni, S. R., Frail, D. A., \& Soderberg, A. M. 2003, ApJ, 599, 408

Berger, E., Zauderer, A., Pooley, G. G., et al. 2012, ApJ, 748, 36

Best, P. N., \& LOFAR-UK Consortium, t. 2008, ArXiv e-prints, arXiv:0802.1186

Blain, A. W., Smail, I., Ivison, R. J., Kneib, J.-P., \& Frayer, D. T. 2002, Phys. Rep., 369, 111

Bloom, J. S., Frail, D. A., \& Kulkarni, S. R. 2003, ApJ, 594, 674

Bloom, J. S., Giannios, D., Metzger, B. D., et al. 2011, Science, 333,203

Booth, R. S., de Blok, W. J. G., Jonas, J. L., \& Fanaroff, B. 2009, ArXiv e-prints, arXiv:0910.2935 [astro-ph.IM]

Bower, G. C., Metzger, B. D., Cenko, S. B., Silverman, J. M., \& Bloom, J. S. 2013, ApJ, 763, 84

Bower, G. C., Saul, D., Bloom, J. S., et al. 2007, ApJ, 666, 346

Brunthaler, A., Menten, K. M., Reid, M. J., et al. 2009, A\&A, 499, L17

Brunthaler, A., Martí-Vidal, I., Menten, K. M., et al. 2010, A\&A, 516, A27

Bucciantini, N., Metzger, B. D., Thompson, T. A., \& Quataert, E. 2012, MNRAS, 419, 1537

Burrows, D. N., et al. 2011, Nature, 476, 421

Carilli, C., \& Rawlings, S. 2004, New Astron. Rev., 48, 979

Carilli, C. L., Ivison, R. J., \& Frail, D. A. 2003, ApJ, 590, 192

Carilli, C. L., \& Yun, M. S. 1999, ApJ, 513, L13

Cendes, Y., et al. 2014, ArXiv e-prints, arXiv:1412.3986 [astro-ph.HE]

Cenko, S. B., Krimm, H. A., Horesh, A., et al. 2012, ApJ, 753, 77

Chandra, P., \& Frail, D. A. 2012, ApJ, 746, 156

Chevalier, R. A. 1982, ApJ, 258, 790

—. 2006, ArXiv Astrophysics e-prints, astro-ph/0607422
Chevalier, R. A., \& Fransson, C. 2006, ApJ, 651, 381

Condon, J. J. 1992, ARA\&A, 30, 575

Condon, J. J., Cotton, W. D., Greisen, E. W., et al. 1998, AJ, 115,1693

Corsi, A., \& Mészáros, P. 2009, ApJ, 702, 1171

Coward, D. M. 2005, MNRAS, 360, L77

Croft, S., Bower, G. C., Keating, G., et al. 2011, ApJ, 731, 34

Croft, S., Bower, G. C., \& Whysong, D. 2013, ApJ, 762, 93

Croft, S., Bower, G. C., Ackermann, R., et al. 2010a, ApJ, 719, 45

Croft, S., et al. 2010b, ApJ, 719, 45

Cucciati, O., Tresse, L., Ilbert, O., et al. 2012, A\&A, 539, A31

de Vries, W. H., Becker, R. H., White, R. L., \& Helfand, D. J. 2004, AJ, 127, 2565

Donnarumma, I., Rossi, E. M., Fender, R., et al. 2015, ArXiv e-prints, arXiv:1501.04640 [astro-ph.HE]

Ellingson, S. W., Clarke, T. E., Cohen, A., et al. 2009, IEEE Proceedings, 97, 1421

Fender, R. 2012, in IAU Symposium, Vol. 285, IAU Symposium, ed. E. Griffin, R. Hanisch, \& R. Seaman, 11

Fender, R. P., Wijers, R. A. M. J., Stappers, B., et al. 2006, in VI Microquasar Workshop: Microquasars and Beyond

Feng, L., Vaulin, R., \& Hewitt, J. N. 2014, MNRAS submitted, arxiv: 1405.6219

Fong, W., \& Berger, E. 2013, ApJ, 776, 18

Frail, D. A., Kulkarni, S. R., Nicastro, L., Feroci, M., \& Taylor, G. B. 1997, Natur, 389, 261

Frail, D. A., Kulkarni, S. R., Ofek, E. O., Bower, G. C., \& Nakar, E. 2012, ApJ, 747, 70

Frail, D. A., Soderberg, A. M., Kulkarni, S. R., et al. 2005, ApJ, 619,994

Frail, D. A., Waxman, E., \& Kulkarni, S. R. 2000, ApJ, 537, 191

Frail, D. A., Kulkarni, S. R., Sari, R., et al. 2001, ApJ, 562, L55

Frail, D. A., Cameron, P. B., Kasliwal, M., et al. 2006, ApJ, 646, L99

Gal-Yam, A., Ofek, E. O., Poznanski, D., et al. 2006, ApJ, 639, 331

Ghirlanda, G., Burlon, D., Ghisellini, G., et al. 2014, PASA, 31, 22

Giannios, D., \& Metzger, B. D. 2011, MNRAS, 416, 2102

Giannios, D., Mimica, P., \& Aloy, M. A. 2008, A\&A, 478, 747

Granot, J., \& Sari, R. 2002, ApJ, 568, 820

Guetta, D., \& Della Valle, M. 2007, ApJ, 657, L73

Hallinan, G., Antonova, A., Doyle, J. G., et al. 2008, ApJ, 684, 644

Hansen, B. M. S., \& Lyutikov, M. 2001, MNRAS, 322, 695 
Hopkins, P. F., Richards, G. T., \& Hernquist, L. 2007, ApJ, 654, 731

Horiuchi, S., Beacom, J. F., Kochanek, C. S., et al. 2011, ApJ, 738,154

Hotokezaka, K., Kiuchi, K., Kyutoku, K., et al. 2013, Phys. Rev. D, 87, 024001

Hotokezaka, K., \& Piran, T. 2015, ArXiv e-prints, arXiv: 1501.01986 [astro-ph.HE]

Jaeger, T., Kassim, N., Hyman, S., et al. 2012a, in American Astronomical Society Meeting Abstracts, Vol. 219, American Astronomical Society Meeting Abstracts \#219, 446.15

Jaeger, T. R., Hyman, S. D., Kassim, N. E., \& Lazio, T. J. W. 2012b, AJ, 143, 96

Jessner, A., Słowikowska, A., Klein, B., et al. 2005, Advances in Space Research, 35, 1166

Johnston, S., Taylor, R., Bailes, M., et al. 2008a, Experimental Astronomy, 22, 151

Johnston, S., et al. 2008b, Experimental Astronomy, 22, 151

Kellermann, K. I., \& Pauliny-Toth, I. I. K. 1969, ApJ, 155, L71

Kim, C., Bhakthi Pranama Perera, B., \& McLaughlin, M. A 2013, ArXiv e-prints, arXiv:1308.4676 [astro-ph.SR]

Kulkarni, S. R., Frail, D. A., Wieringa, M. H., et al. 1998, Nature, 395, 663

Kulkarni, S. R., Frail, D. A., Sari, R., et al. 1999, ApJ, 522, L97

Lane, W. M., Cotton, W. D., Helmboldt, J. F., \& Kassim, N. E. 2012, Radio Science, 47, 0

Laskar, T., Berger, E., Zauderer, B. A., et al. 2013, ApJ, 776, 119

Leibler, C. N., \& Berger, E. 2010, ApJ, 725, 1202

Levan, A. J., et al. 2011, Science, 333, 199

Levinson, A., Ofek, E. O., Waxman, E., \& Gal-Yam, A. 2002, ApJ, 576, 923

Lonsdale, C. J., Cappallo, R. J., Morales, M. F., et al. 2009, IEEE Proceedings, 97, 1497

Lyubarsky, Y. 2014, MNRAS, 442, L9

Macquart, J.-P. 2014, PASA, 31, 31

Matzner, C. D., \& McKee, C. F. 1999, ApJ, 510, 379

Mészáros, P., \& Rees, M. J. 1997, ApJ, 476, 232

Metzger, B. D., \& Berger, E. 2012, ApJ, 746, 48

Metzger, B. D., \& Bower, G. C. 2014, MNRAS, 437, 1821

Metzger, B. D., Giannios, D., \& Mimica, P. 2012, MNRAS, 420, 3528

Metzger, B. D., \& Piro, A. L. 2014, MNRAS, 439, 3916

Metzger, B. D., Quataert, E., \& Thompson, T. A. 2008, MNRAS, 385,1455

Mimica, P., Giannios, D., Metzger, B. D., \& Aloy, M. A. 2015, ArXiv e-prints, arXiv:1501.00361 [astro-ph.HE]

Mooley, K. P., Frail, D. A., Ofek, E. O., et al. 2013, ApJ, 768, 165

Murphy, T., et al. 2013, PASA, 30, 6

Nakar, E., \& Piran, T. 2011, Nature, 478, 82

Nakar, E., \& Sari, R. 2012, ApJ, 747, 88

Ofek, E. O., Breslauer, B., Gal-Yam, A., et al. 2010, ApJ, 711, 517

Ofek, E. O., Frail, D. A., Breslauer, B., et al. 2011, ApJ, 740, 65

Oosterloo, T., Verheijen, M. A. W., van Cappellen, W., et al. 2009, in Wide Field Astronomy \& Technology for the Square Kilometre Array
Perley, R. A., Chandler, C. J., Butler, B. J., \& Wrobel, J. M. 2011, ApJ, 739, L1

Pietka, M., Fender, R. P., \& Keane, E. F. 2015, MNRAS, 446, 3687

Piro, A. L., \& Kulkarni, S. R. 2013, ApJ, 762, L17

Rosswog, S., Piran, T., \& Nakar, E. 2013, MNRAS, 430, 2585

Sari, R., \& Piran, T. 1999, ApJ, 520, 641

Sari, R., Piran, T., \& Narayan, R. 1998, ApJ, 497, L17

Shivvers, I., \& Berger, E. 2011, ApJ, 734, 58

Siegel, D. M., Ciolfi, R., \& Rezzolla, L. 2014, ApJ, 785, L6

Sijacki, D., Vogelsberger, M., Genel, S., et al. 2014, ArXiv e-prints, arXiv: 1408.6842

Soderberg, A. M., Kulkarni, S. R., Berger, E., et al. 2005, ApJ, 621,908

- 2004, Nature, 430, 648

Soderberg, A. M., et al. 2008, Nature, 453, 469

-. 2010, Nature, 463, 513

Spitler, L. G., Cordes, J. M., Hessels, J. W. T., et al. 2014, ArXiv e-prints, arXiv:1404.2934 [astro-ph.HE]

Stone, N. C., \& Metzger, B. D. 2014, ArXiv e-prints, arXiv:1410.7772 [astro-ph.HE]

Thornton, D., Stappers, B., Bailes, M., et al. 2013, Science, 341, 53

Usov, V. V. 1992, Nature, 357, 472

van Eerten, H., Zhang, W., \& MacFadyen, A. 2010, ApJ, 722, 235

van Eerten, H. J., \& MacFadyen, A. I. 2011, ApJ, 733, L37

van Haarlem, M. P., et al. 2013, A\&A, 556, A2

van Velzen, S., Frail, D. A., Körding, E., \& Falcke, H. 2013, A\&A, 552 , A5

van Velzen, S., Körding, E., \& Falcke, H. 2011, MNRAS, 417, L51

Wanderman, D., \& Piran, T. 2010, MNRAS, 406, 1944

-. 2014, ArXiv e-prints, arXiv:1405.5878 [astro-ph.HE]

Wang, J., \& Merritt, D. 2004, ApJ, 600, 149

Weiler, K. W., Panagia, N., Montes, M. J., \& Sramek, R. A. 2002, ARA\&A, 40, 387

Williams, P. K. G., \& Bower, G. C. 2010, ApJ, 710, 1462

Williams, P. K. G., Bower, G. C., Croft, S., et al. 2013, ApJ, 762, 85

Wygoda, N., Waxman, E., \& Frail, D. A. 2011, ApJ, 738, L23

Yin, Q. F., \& Heeschen, D. S. 1991, Nature, 354, 130

Yu, Y.-W., Zhang, B., \& Gao, H. 2013, ApJ, 776, L40

Yüksel, H., Kistler, M. D., Beacom, J. F., \& Hopkins, A. M. 2008, ApJ, 683, L5

Yungelson, L., \& Livio, M. 1998, ApJ, 497, 168

Zauderer, B. A., Berger, E., Margutti, R., et al. 2013, ApJ, 767, 152

Zauderer, B. A., et al. 2011, Nature, 476, 425

Zhang, W., \& MacFadyen, A. 2009, ApJ, 698, 1261 